\title{
Image-Based Cardiac Diagnosis With Machine Learning: A Review
}

\author{
Carlos Martin-Isla ${ }^{1 *}$, Victor M. Campello ${ }^{1}$, Cristian Izquierdo ${ }^{1}$, Zahra Raisi-Estabragh ${ }^{2,3}$, \\ Bettina Baeßler ${ }^{4}$, Steffen E. Petersen ${ }^{2,3}$ and Karim Lekadir ${ }^{1}$ \\ ${ }^{1}$ Departament de Matemàtiques \& Informàtica, Universitat de Barcelona, Barcelona, Spain, ${ }^{2}$ Barts Heart Centre, Barts \\ Health NHS Trust, London, United Kingdom, ${ }^{3}$ William Harvey Research Institute, Queen Mary University of London, London, \\ United Kingdom, ${ }^{4}$ Department of Diagnostic \& Interventional Radiology, University Hospital Zurich, Zurich, Switzerland
}

OPEN ACCESS

Edited by:

Sebastian Kelle,

Deutsches Herzzentrum Berlin,

Germany

Reviewed by:

Joao Bicho Augusto,

Barts Heart Centre, United Kingdom

John Hoe,

MediRad Associates Ltd, Singapore

*Correspondence:

Carlos Martin-Isla

carlos.martinisla@ub.edu

Specialty section:

This article was submitted to

Cardiovascular Imaging,

a section of the journal

Frontiers in Cardiovascular Medicine

Received: 01 November 2019 Accepted: 06 January 2020

Published: 24 January 2020

Citation:

Martin-Isla C, Campello VM Izquierdo C, Raisi-Estabragh Z,

Baeßler B, Petersen SE and Lekadir K

(2020) Image-Based Cardiac

Diagnosis With Machine Learning: A

Review. Front. Cardiovasc. Med. 7:1.

doi: 10.3389/fcvm.2020.00001
Cardiac imaging plays an important role in the diagnosis of cardiovascular disease (CVD). Until now, its role has been limited to visual and quantitative assessment of cardiac structure and function. However, with the advent of big data and machine learning, new opportunities are emerging to build artificial intelligence tools that will directly assist the clinician in the diagnosis of CVDs. This paper presents a thorough review of recent works in this field and provide the reader with a detailed presentation of the machine learning methods that can be further exploited to enable more automated, precise and early diagnosis of most CVDs.

Keywords: cardiovascular disease, automated diagnosis, cardiac imaging, artificial intelligence, machine learning, deep learning, radiomics

\section{INTRODUCTION}

Despite significant advances in diagnosis and treatment, cardiovascular disease (CVD) remains the most common cause of morbidity and mortality worldwide, accounting for approximately one third of annual deaths $(1,2)$. Early and accurate diagnosis is key to improving CVD outcomes. Cardiovascular imaging has a pivotal role in diagnostic decision making. Current image analysis techniques are mostly reliant on qualitative visual assessment of images and crude quantitative measures of cardiac structure and function. In order to optimize the diagnostic value 5 of cardiac imaging, there is need for more advanced image analysis techniques that allow deeper quantification of imaging phenotypes. In recent years, the development of big data and availability of high computational power have driven exponential advancement of artificial intelligence (AI) technologies in medical imaging (Figure 1). Machine learning (ML) approaches to image-based diagnosis rely on algorithms/models that learn from past clinical examples through identification of hidden and complex imaging patterns. Existing work already demonstrates the incremental value of image-based cardiovascular diagnosis with ML for a number of important conditions such as coronary artery disease (CAD) and heart failure (HF). The superior diagnostic performance of $\mathrm{AI}$ image analysis has the potential to substantially alleviate the burden of cardiovascular disease through facilitation of faster and more accurate diagnostic decision making.

In this paper we describe the main ML techniques and the procedures required to successfully design, implement, and validate new ML tools for image-based diagnosis. We also present a comprehensive review of existing literature pertaining to applications of ML for image-based diagnosis of CVD. 


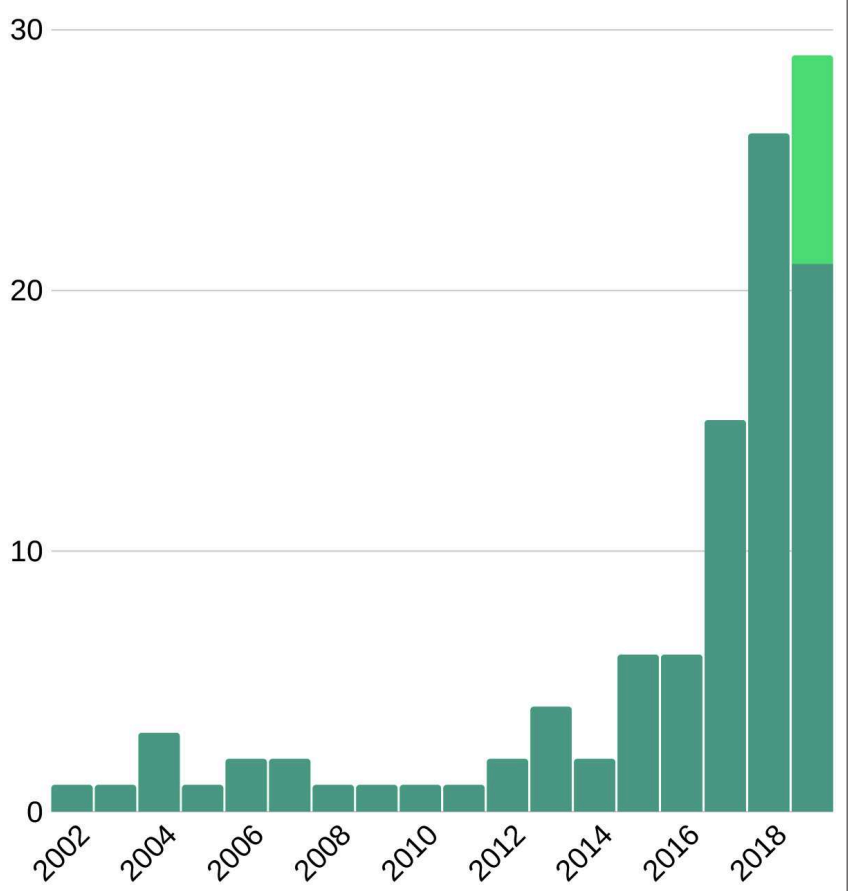

FIGURE 1 | Number of publications on machine learning and cardiac imaging per year. This suggests an upward trend for future research. Light green bar represents the expected number of publications to be published late 2019 .

\section{OVERVIEW OF PIPELINE FOR IMAGE-BASED MACHINE LEARNING DIAGNOSIS}

The overall pipeline to build ML tools for image-based cardiac diagnosis is schematically described in the following section, as well as in Figure 2. In short, it requires (1) input imaging datasets from which suitable imaging predictors can be extracted, (2) accurate output diagnosis labels, and (3) a suitable ML technique that is typically chosen and optimized depending

Abbreviations: Machine learning abbreviations: AI, Articial Intelligence; AUC, Area Under Curve; ANN, Artificial Neural Networks; BN, Bayesian Network; CNN, Convolutional Neural Network; CL, Clustering; DL, Deep Learning; DT, Decision Tree; GA, Genetic Algorithm; GAN, Generative Adversarial Network; GBRT, Gradient Boosting Trees; kNN, k-Nearest Neighbors; LDA, Linear Discriminant Analysis; LR, Logistic Regression; ML, Machine Learning; PCA, Principal Component Analysis; PLS, Partial Least Squares; RF, Random Forest; ROC, Receiver Operating Characteristic Curve; Se, Sensitivity; Sp, Specificity; SVM, Support Vector Machine; VAE, Variational Autoencoder.

Cardiac imaging and clinical abbreviations: ARV, Abnormal Right Ventricle; ASD, Atrial Septal Defect; CAC, Coronary Artery Calcium; CAD, Coronary Artery Disease; CMR, Cardiac Magnetic Resonance; CT, Computed Tomography; CTA, Computed Tomography Angiography; CVD, Cardiovascular Disease; DCM, Dilated Cardiomyopathy; ECG, Electrocardiography; echo, Echocardiography; HCM, Hypertrophic Cardiomyopathy; HF, Heart Failure; HFpEF, Heart Failure with preserved Ejection Fraction; HHD, Hypertensive Heart Disease; ICA, Invasive Coronary Angiography; IR, Iterative Reconstruction; LV, Left Ventricle; MACE, Major Adverse Cardiovascular Event; MI, Myocardial Infarction; MR, Mitral Regurgitation; MRI, Magnetic Resonance Imaging; MYO, Myocarditis; NRS, Napkin Ring Sign; PET, Positron Emission Tomography; ROI, Region Of Interest; RV, Right Ventricle; SPECT, Single Positron Emission Computed Tomography. on the application to predict the cardiac diagnosis (output) based on the imaging predictors (input). Additional non-imaging predictors (e.g., electrocardiogram data, genetic data, sex, or age) are often integrated into the ML model and typically improve model performance.

In this section, we will first discuss the input and output variables in more detail, before introducing common used ML techniques and their applications.

\subsection{Data, Input and Output Variables}

\subsubsection{Sources of Cardiovascular Imaging Data}

Robust ML models are reliant on the availability of sufficient and accurate data. Thus, data preparation is an important pre-requisite to derive that perform well on internal and external validation. Within cardiac imaging, there is increasing availability of quality sources of organized big data through various biobanks, bioresources, and registries. Available cohorts can be classified into population-based and clinical cohorts. Population cohorts such as the UK Biobank follow the health status of a representative sample of individuals from the general population and thus are particularly useful for risk stratification. In contrast, clinical cohorts, such as the Barts BioResource or the European cardiovascular magnetic resonance (EuroCMR) registry, are composed of clinical imaging from patients and therefore more suitable for building diagnostic tools. These datasets are an invaluable resource for the development and validation of ML diagnostic models (see Table 1 for examples of additional cardiac imaging datasets).

\subsubsection{Input Variables}

Before an ML model can be built for image-based diagnosis estimation, it is necessary to suitably define the imaging inputs. Imaging inputs may be the raw imaging data (i.e., pixel intensities), conventional cardiac indices (and other transformed quantitative image parameters) or radiomics features extracted from the image. See Figures $\mathbf{3}$ and $\mathbf{4}$ for additional information about input variables.

\subsubsection{Conventional imaging indices}

Conventional imaging indices include measures commonly used in routine clinical image analysis such as ventricular volumes in end diastole/systole and ventricular ejection fractions.

Estimation of these clinical indices requires prior contouring of the endocardial and epicardial boundaries of the relevant cardiac chambers. Deep learning approaches have been used to develop automated/semi-automated contouring tools for more efficient and reproducible segmentation of cardiac chambers.

Since manual delineation of these boundaries is tedious and subject to errors, many automatic or semi-automatic tools have been developed (see Table 2 for examples of existing tools). Note that recently, many deep learning (DL) based approaches have been published for accurate and robust segmentation of the cardiac boundaries with promising results, however this is beyond the scope of this review [more details on this, as well as a basic introduction to $\mathrm{ML}$, in cardiac magnetic resonance imaging (MRI) can be found in recent work by (3)]. 


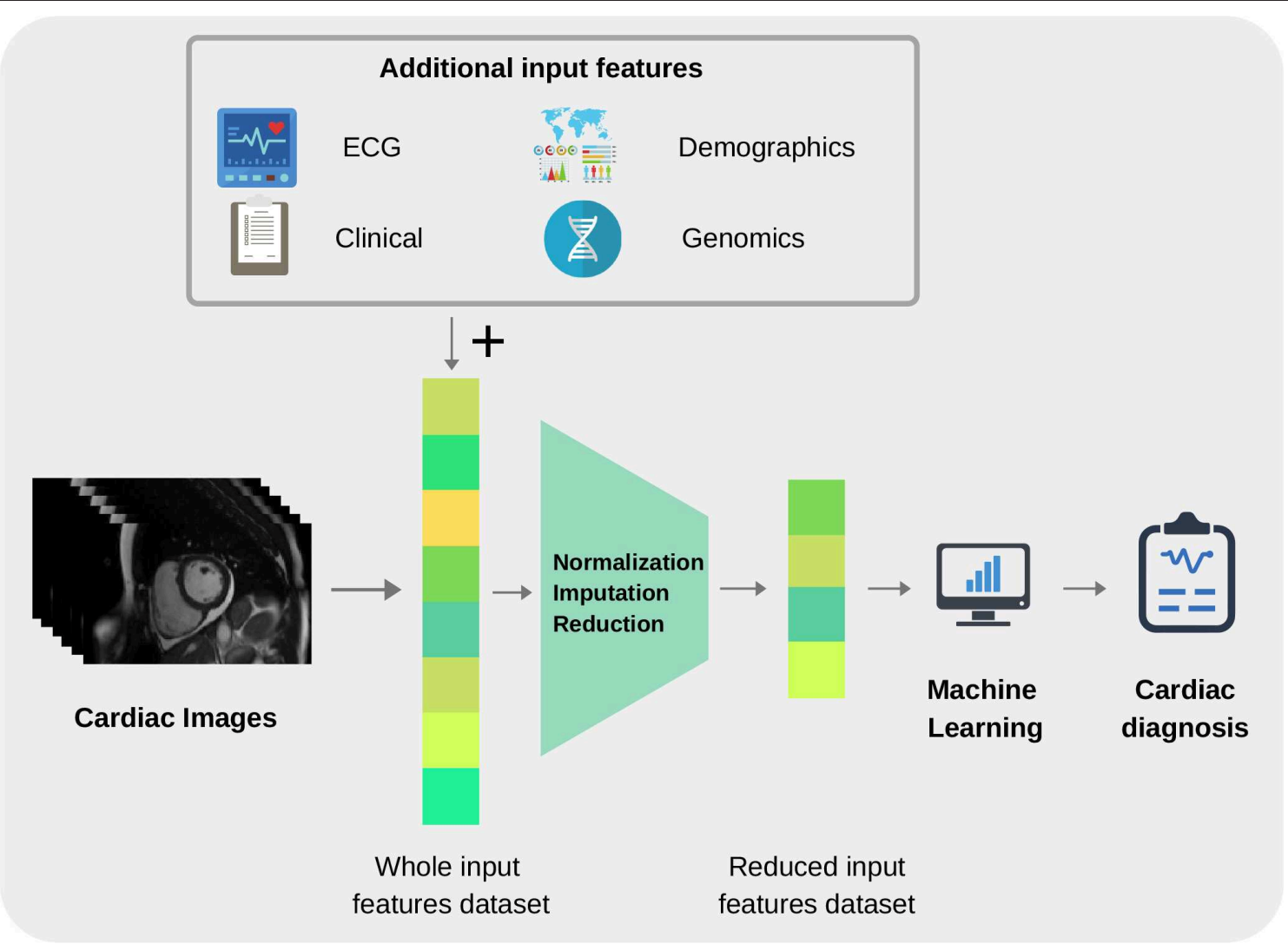

FIGURE 2 | Pipeline for building image-based machine learning models.

TABLE 1 | Selection of cardiac imaging datasets available.

\begin{tabular}{|c|c|c|c|c|}
\hline Name & Country & Modality & Size & Year \\
\hline Framingham Heart Study & USA & Echo/MRI/CT & $>5,000$ & 1948 \\
\hline Study of Health in Pomerania & DE & $\mathrm{MRI}$ & $>8,000$ & 1997 \\
\hline The Tromso Study & NO & Echo & 3,287 & 1999 \\
\hline Multi-Ethnic Study of Atherosclerosis & USA & $\mathrm{MRI}$ & 2,450 & 2000 \\
\hline UK Biobank & UK & $\mathrm{MRI}$ & 20,000 & 2006 \\
\hline HUNT Study & NO & Echo & 1,296 & 2006 \\
\hline Defibrillators to Reduce Risk by MRI Evaluation & USA & $\mathrm{MRI}$ & 450 & 2007 \\
\hline Barts BioResource & UK & Echo / MRI & $>10,000$ & 2007 \\
\hline European CMR Registry & EU & $\mathrm{MRI}$ & $>27,000$ & 2007 \\
\hline NEO Study & $\mathrm{NL}$ & $\mathrm{MRI}$ & 1,205 & 2008 \\
\hline SunnyBrook Cardiac Data & USA & $\mathrm{MRI}$ & 45 & 2009 \\
\hline Registry of Fast Myocardial Perfusion Imaging with next gen SPECT & USA & SPECT & $>20,400$ & 2009 \\
\hline The German National Cohort & $\mathrm{DE}$ & $\mathrm{MRI}$ & 20,000 & 2011 \\
\hline Maastricht Study & $\mathrm{NL}$ & Echo / CT & 3,451 & 2012 \\
\hline Canadian Alliance for Healthy Hearts and Minds & CA & $\mathrm{MRI}$ & 9,700 & 2013 \\
\hline Challenge on Endocardial 3D Ultrasound Segmentation & FR & Echo & 45 & 2014 \\
\hline Hamburg City Health Study & $\mathrm{DE}$ & $\mathrm{MRI}$ & $>45,000$ & 2016 \\
\hline Automated Cardiac Diagnosis Challenge Dataset & $\mathrm{FR}$ & $\mathrm{MRI}$ & 150 & 2017 \\
\hline Cardiac Acquisitions for Multi-structure Ultrasound Segmentation & $\mathrm{FR}$ & Echo & 500 & 2019 \\
\hline
\end{tabular}



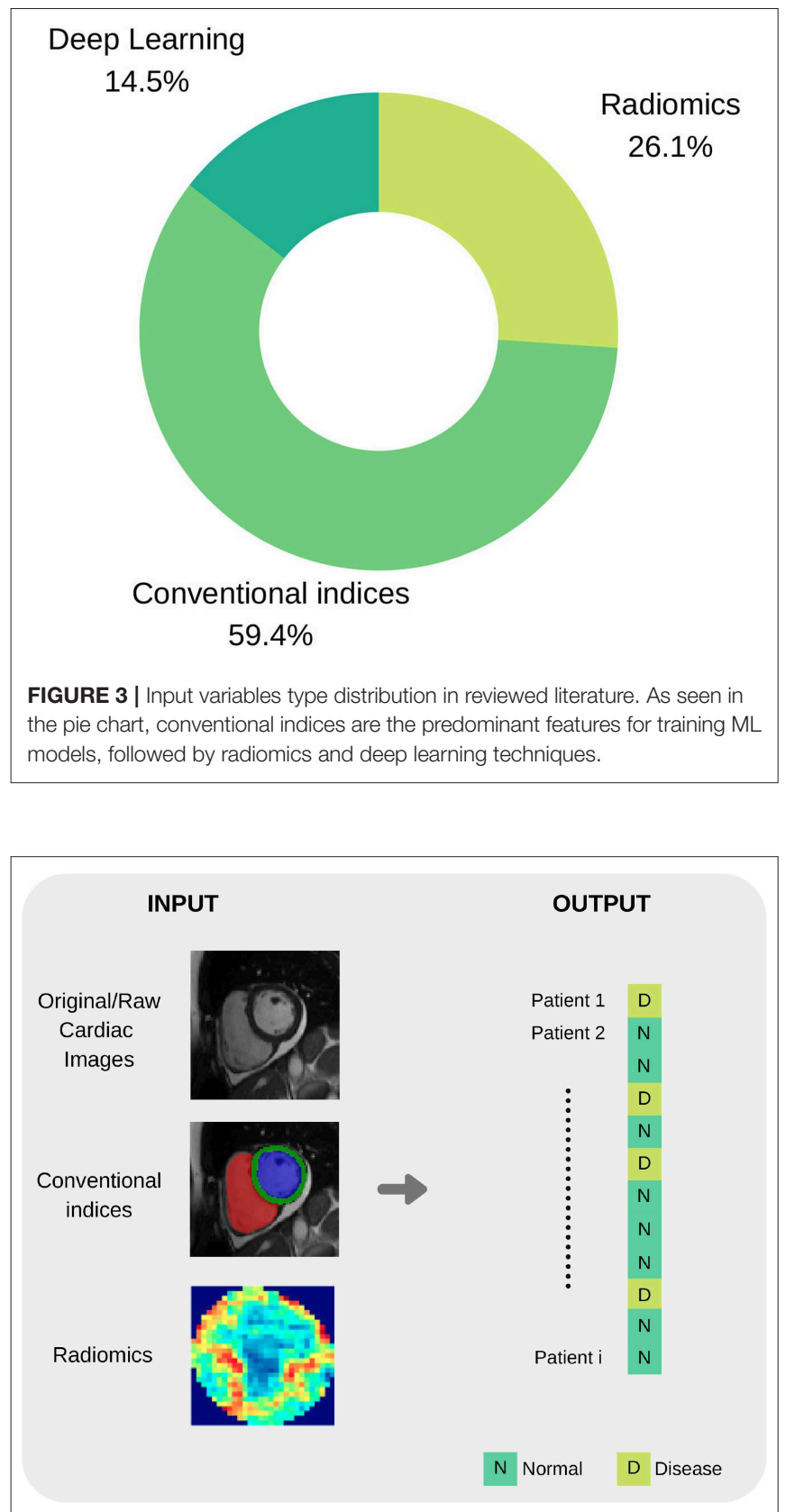

FIGURE 4 | Summary of common input and output variables for image-based diagnosis $\mathrm{ML}$ algorithms. Different cardiac imaging input features such as raw data, conventional indices extracted from a $\mathrm{ROI}$ or radiomics (delineation of cardiac anatomy is required for the last two cases) and desired output. Both structures shape the most basic requirement for a $\mathrm{ML}$ cardiac imaging application, data.

Some recent works will be listed to illustrate the use of conventional imaging indices as inputs for ML-based diagnosis models. In Khened et al. (4), an artificial neural network (ANN) was built to automatically diagnose several cardiac diseases such as hypertrophic cardiomyopathy (HCM), myocardial infarction (MI) and abnormal RV (ARV), by using as input LV and RV
TABLE 2 | Selection of cardiac structural and functional analysis softwares.

\begin{tabular}{lll}
\hline Name & Producer & Modality \\
\hline CMRtools & Cardiovascular Imaging Solutions & MRI \\
suiteHEART & NeoSoft & MRI \\
CVI42 & Circle Cardiovascular Imaging & MRI/CT \\
Medis Suite & Medis & MRI/CT \\
iNtuition & Terarecon & MRI/CT \\
Segment & Medviso & MRI/CT/SPECT \\
syngo.via & Siemens & MRI/CT/SPECT \\
IntelliSpace Portal & Philips & MRI/CT/echo \\
VevoLAB & Visualsonics & Echo \\
QLAB & Philips & Echo \\
TOMTEC & Philips & Echo \\
\hline
\end{tabular}

ejection fraction, RV and LV volume end-systole and enddiastole, myocardial mass, as well as the patient's height and weight. In Chen et al. (5), the authors integrated a set of 32 variables from clinical data, including ejection fraction, blood pressure, sex, age, as well as other conventional risk factors, to diagnose dilated cardiomyopathy (DCM). Juarez-Orozco et al. (6) merged ejection fractions at rest and stress with a pool of clinical parameters to predict ischemia and adverse cardiovascular events using ML.

Regarding motion, strain and single intensity analysis, in Mantilla et al. (7), global spatio-temporal image features are extracted to feed a support vector machine (SVM) classifier for LV wall motion assessment. Pairwise single intensity and variance regional differences in SPECT perfusion studies mimics the clinical procedure of qualitatively comparing stress and rest images in Bagher-Ebadian et al. (8). Contractility differences and multiscale wall motion assessment are performed by means of apparent flow in Moreno et al. (9) and Zheng et al. (10) where each feature describes an oriented velocity at a given position along the cardiac ROI.

\subsubsection{Radiomics features}

Radiomics analysis is the process of converting digital images to minable data. Analysis of the data through application of various statistical and mathematical processes allows quantification of various shape and textural characteristics of the image, referred to as radiomics features (Table 3 ). Radiomics analysis quantifies more advanced and complex characteristics of the cardiac chambers than is visually perceptible. Similarly to clinical imaging indices, radiomics requires the delineation of the cardiac structures before the features can be extracted.

Introduced in $2012(11,12)$, the radiomics paradigm was, for a long time, mostly exploited in oncology (13). Recently, a number of works have shown the promise of radiomics combined with ML for image-aided diagnosis of CVD. For instance, Cetin et al. (14) demonstrated that about 10 radiomics features integrated within an ML model are sufficient to discriminate between several major CVDs. More recently, researchers at Harvard University, Neisius et al. (15) have built an ML model with 6 radiomic features calculated from T1 mapping 
TABLE 3 | Radiomics features overview.

\begin{tabular}{|c|c|c|}
\hline Type & Description & Examples \\
\hline Shape features & $\begin{array}{l}\text { Describe geometric } \\
\text { characteristics of the cardiac } \\
\text { structures }\end{array}$ & $\begin{array}{l}\text { Volume, surface area, } \\
\text { sphericity, } \\
\text { diameters, axis, surface } \\
\text { to volume ratio, flatness }\end{array}$ \\
\hline $\begin{array}{l}\text { Intensity (First } \\
\text { order) }\end{array}$ & $\begin{array}{l}\text { Statistics on the intensity } \\
\text { distributions within the region of } \\
\text { interest }(\mathrm{ROI})\end{array}$ & $\begin{array}{l}\text { Mean intensity, range, } \\
\text { skewness } \\
\text { (asymmetry) and } \\
\text { entropy }\end{array}$ \\
\hline $\begin{array}{l}\text { Texture GLCM } \\
\text { (Second order) }\end{array}$ & $\begin{array}{l}\text { Quantifies the spatial relationship } \\
\text { of the pixels in the ROI }\end{array}$ & Contrast, correlation \\
\hline $\begin{array}{l}\text { Texture GLSZM } \\
\text { (Higher order) }\end{array}$ & $\begin{array}{l}\text { Quantifies the number of } \\
\text { connected voxels that share the } \\
\text { same intensity level }\end{array}$ & $\begin{array}{l}\text { Gray level } \\
\text { non-uniformity, zone } \\
\text { entropy }\end{array}$ \\
\hline $\begin{array}{l}\text { Texture GLRLM } \\
\text { (Higher order) }\end{array}$ & $\begin{array}{l}\text { Quantifies the gray level runs in } \\
\text { the ROI }\end{array}$ & $\begin{array}{l}\text { Run entropy, long run } \\
\text { emphasis } \\
\text { and short run emphasis }\end{array}$ \\
\hline $\begin{array}{l}\text { Texture NGTDM } \\
\text { (Higher order) }\end{array}$ & $\begin{array}{l}\text { Quantifies the difference between } \\
\text { a gray value and the average gray } \\
\text { value of its neighbors within a } \\
\text { predefined distance }\end{array}$ & Busyness, strength \\
\hline $\begin{array}{l}\text { Texture GLDM } \\
\text { (Higher order) }\end{array}$ & $\begin{array}{l}\text { Quantifies the gray level } \\
\text { dependencies in the ROI }\end{array}$ & $\begin{array}{l}\text { Dependence non- } \\
\text { uniformity, } \\
\text { dependence entropy } \\
\text { and } \\
\text { dependence variance }\end{array}$ \\
\hline Fractal dimension & $\begin{array}{l}\text { Determines the ratio of change in } \\
\text { detail to the change in scale }\end{array}$ & \\
\hline
\end{tabular}

sequences to differentiate between hypertensive heart disease (HHD) and HCM.

\subsubsection{Raw imaging data}

Whole raw images may also be used as the input for the ML model, without any pre-processing or calculation of hand-crafted input imaging features. About $10 \%$ of published reports rely on this type of modeling. In this case, the optimal features for predicting the cardiac diagnoses are selflearned automatically by the ML techniques based on the training sample, as opposed to a priori definition by the AI scientist.

For illustration, it is worth mentioning the work by Betancur et al. (16), an end-to-end DL model, estimating per-vessel CAD probability without any assumed subdivision of the input coronary territories from imaging data. The authors in Wolterink et al. (17) built a coronary artery calcification (CAC) detector, also based on DL trained on raw CT images. A similar DL model directly built from raw echo images was demonstrated in $\mathrm{Lu}$ et al. (18) to identify dilated cardiomyopathy cases. Also from raw echo images, the authors in Kusunose et al. (19) built a DL model for automatic detection of regional wall motion abnormalities.

\subsubsection{Output}

ML algorithms may be developed using supervised or unsupervised learning methods. Supervised learning requires accurately labeled training examples. In the simplest form, the output is a binary variable which takes a value of 1 for a diseased individual and 0 for a control healthy subject. To obtain a robust ML model, it is recommended to use a balanced training sample, comprising a similar number of healthy and diseased subjects. Note that the binary classification can be easily extended to the multi-class case if several diseases or stages of disease are to be included in the ML model. Thus, supervised learning algorithms link the input variables to labeled outputs. Unsupervised learning is the training of algorithms without definition of the output. Through this technique, the ML algorithm groups the sample through recognition of inherent patterns within the data. In general, supervised learning outperforms unsupervised learning and so is the preferred method in situations where the ground truth is known. However, unsupervised learning has unique value for discovery of novel disease sub-types and patient stratification e.g., different pheno-groups of hypertensive heart disease or CAD.

\subsection{Machine Learning Techniques}

ML, refers to the use of computer algorithms that have the capacity to learn to perform given tasks from example data without the need for explicitly programmed instructions, i.e., image-based cardiac diagnosis in our case. This field of AI uses advanced statistical techniques to extract predictive or discriminatory patterns from the training data in order to perform the most accurate predictions on new data. We present the most commonly used ML techniques in the field of cardiac imaging and diagnosis for a non-expert audience and discuss their benefits and drawbacks (see Table 4 and Figure 5 for additional information). A list of diagnostic applications for each method will be provided as examples.

\subsubsection{Logistic Regression}

A Logistic Regression (LR) model is used to estimate the probability of a given output based on input variables in a continuous fashion, in contrast with a binary classifier. Final probabilities add up to one, so one obtains a stratification into all possible outcomes and the odds for each one. One property of this model is that a slight change in the input value may disproportionately impact the final probability prediction, as can be seen in Figure 6A. Additionally, the input vector dimension (number of predictor variables) must be kept low, as this can lead to costly model training processes and risks overfitting of the model to the training dataset with resultant poor generalisability of the model. Thus, when dealing with a large number of input variables, dimensionality reduction algorithms, such as principal component analysis (PCA) or linear discriminant analysis (LDA), are applied to reduce the number of predictors to those that are most informative. LR is a valuable model to be selected when different sources of data must be integrated in a binary classification task and low complexity is required.

In the literature, several works have applied LRs for their particular application. For example, Zheng et al. (10) applied a sequence of four LRs to classify patients according to cardiac pathologies by using shape features extracted from cine MRI 
TABLE 4 | Overview of machine learning techniques.

\begin{tabular}{|c|c|c|c|}
\hline Technique & Description & Advantages & Disadvantages \\
\hline $\begin{array}{l}\text { Logistic } \\
\text { Regression }\end{array}$ & $\begin{array}{l}\text { Extension of linear } \\
\text { regression that } \\
\text { outputs a } \\
\text { binary } \\
\text { classification }\end{array}$ & $\begin{array}{l}\text { Simple and } \\
\text { explainable; } \\
\text { Does not require } \\
\text { empirical parameter } \\
\text { tuning nor input } \\
\text { feature } \\
\text { normalization }\end{array}$ & $\begin{array}{l}\text { Not suitable for non- } \\
\text { linear problems; } \\
\text { Prone to } \\
\text { overfitting }\end{array}$ \\
\hline $\begin{array}{l}\text { Support } \\
\text { Vector } \\
\text { Machine }\end{array}$ & $\begin{array}{l}\text { Finds the optimal } \\
\text { boundary between } \\
\text { classes }\end{array}$ & $\begin{array}{l}\text { Can handle different } \\
\text { types of non-linear } \\
\text { class } \\
\text { separations; Does } \\
\text { not } \\
\text { require large training } \\
\text { samples }\end{array}$ & $\begin{array}{l}\text { Requires } \\
\text { hyperparameter } \\
\text { tuning and } \\
\text { non-linear } \\
\text { kernel selection; Not } \\
\text { suitable for very } \\
\text { large } \\
\text { datasets }\end{array}$ \\
\hline $\begin{array}{l}\text { Random } \\
\text { Forest }\end{array}$ & $\begin{array}{l}\text { Generates a set of } \\
\text { hierarchical } \\
\text { decision } \\
\text { queries over the } \\
\text { input } \\
\text { and output data }\end{array}$ & $\begin{array}{l}\text { Automatically } \\
\text { defines } \\
\text { feature importances; } \\
\text { Does not require } \\
\text { input } \\
\text { feature normalization }\end{array}$ & $\begin{array}{l}\text { Prone to overfitting; } \\
\text { Requires definition of } \\
\text { depth and number } \\
\text { of } \\
\text { trees }\end{array}$ \\
\hline $\begin{array}{l}\text { Artificial } \\
\text { Neural } \\
\text { Network }\end{array}$ & $\begin{array}{l}\text { Models complex } \\
\text { classification tasks } \\
\text { by propagating } \\
\text { input data through } \\
\text { a } \\
\text { network of } \\
\text { non-linear } \\
\text { transformations }\end{array}$ & $\begin{array}{l}\text { Generalizes well } \\
\text { when } \\
\text { trained on large } \\
\text { training } \\
\text { samples }\end{array}$ & $\begin{array}{l}\text { Difficult to interpret; } \\
\text { Requires prior } \\
\text { selection } \\
\text { of a network design } \\
\text { (e.g., } \\
\text { depth of the } \\
\text { network); } \\
\text { Requires large } \\
\text { training } \\
\text { data }\end{array}$ \\
\hline $\begin{array}{l}\text { Convolutional } \\
\text { Neural } \\
\text { Network }\end{array}$ & $\begin{array}{l}\text { ANNs adjusted for } \\
\text { the processing } \\
\text { and } \\
\text { classification of } \\
\text { image } \\
\text { data }\end{array}$ & $\begin{array}{l}\text { Flexible design } \\
\text { depending on the } \\
\text { applications; Can } \\
\text { learn } \\
\text { the optimal features } \\
\text { directly from the } \\
\text { images }\end{array}$ & $\begin{array}{l}\text { Same limitations as } \\
\text { ANNs }\end{array}$ \\
\hline Clustering & $\begin{array}{l}\text { Finds subgroups } \\
\text { within } \\
\text { the input feature } \\
\text { space in } \\
\text { an unsupervised } \\
\text { manner }\end{array}$ & $\begin{array}{l}\text { Useful to discover } \\
\text { subgroups when the } \\
\text { groups labels are not } \\
\text { unknown a priori; } \\
\text { Simple and fast }\end{array}$ & $\begin{array}{l}\text { Sensitive to } \\
\text { initialization } \\
\text { and scale; Difficult to } \\
\text { estimate the number } \\
\text { of } \\
\text { subgroups }\end{array}$ \\
\hline
\end{tabular}

per segment. Thus they obtained a simple and easily interpretable model with only three input features per classifier. In another example, Arsanjani et al. (20) used a combination of classifiers improved with a LR to diagnose obstructive CAD using SPECT images. Finally, a LR was also applied by Baeßler et al. (21) to diagnose acute or chronic heart failure-like myocarditis.

\subsubsection{Support Vector Machine (SVM)}

Support vector machines (SVMs) are supervised ML models whereby the optimal linear or non-linear boundary segregating the data into two or more classes is identified, as can be seen in Figure 6B. Prior to application of SVMs, the function which will be used for segregating the data should be selected, the so called kernel function. The most used kernels are the linear function or the Gaussian function. The remaining parameters of the SVM model are chosen empirically by training a set of models and keeping the settings as for the model with the lowest error. Since this model is insensitive to non-discriminating dimensions, a dimension reduction could be applied to the input variables to ease the training and obtain a better generalization as for linear regression. One major drawback of SVM is that it becomes memory expensive when large amounts of data are processed. SVM is a good choice to identify non-linearity and sparsity in the input data : different kernels can be used to fit different distributions.

Amongst all ML methods presented in this review, SVM is one of the most frequently used techniques and some works find this model to obtain the best performance. For example, Conforti and Guido (22) presented a comparison of SVM models with different kernels (polynomial, Gaussian and Laplacian functions), the original 105 features and a feature selection of 25 as input for the early diagnosis of myocardial infarction. Similarly, Arsanjani et al. (23) and Ciecholewski (24) found that a SVM model outperformed previous algorithms used in the task of CAD identification by using data extracted from SPECT images. In the first example, a second degree polynomial was used as kernel while in the second, a Gaussian function showed better performance. A SVM was also the best model when predicting acute coronary syndrome for 228 patients using histological, ECG and echo qualitative features, as shown by Berikol et al. (25). As a final example, Borkar and Annadate (26) obtained a very good accuracy for discrimination of DCM and atrial septal defect (ASD) patients using radiomics features and a SVM using a Gaussian kernel function.

\subsubsection{Random Forest (RF)}

This popular technique consists of a combination of decision trees (DTs) trained on different random samples of the training set, as can be seen in Figure 6C. Each DT is a set of rules based on the input features values optimized for accurately classifying all elements of the training set. DTs are nonlinear models and tend to have high variance. If the DT is grown very deep it can pick up irregularities in the training dataset and consequently problems with overfitting may be encountered. This problem is counteracted in a RF through training on different samples of the training dataset. In this way the variance is reduced as the number of DT used, lowering therefore the generalization error and becoming a powerful technique. The final prediction is obtained by selecting the mode (for classification problems) or the mean (for regression problems) of all predictions. Two parameters must be selected for these models: the number of DTs and the depth level for each DT (i.e., the number of decisions). However, one must bear in mind that whilst discriminatory power on training dataset is increased as DT increase in depth, this is often at the expense of losing generalization power. RFs are chosen in order to transform the problem into a set of hierarchical queries represented as DTs. However, RFs are not very resistant to noise.

In the literature, RF or DT have been used frequently and were selected as the best performing model in some works. For 


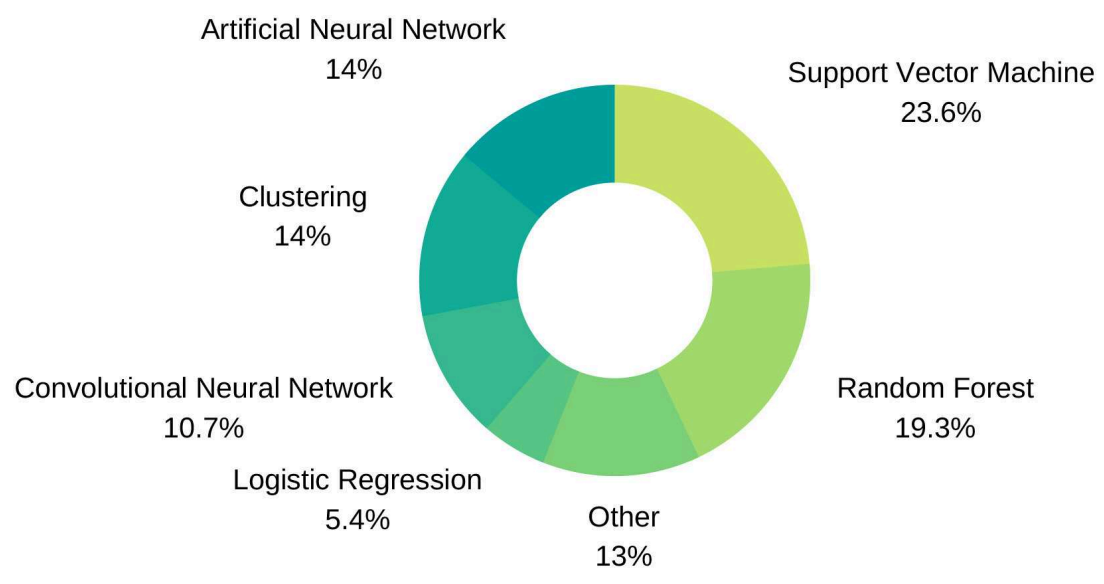

Artificial Neural Network
$23.6 \%$

dom Forest

$19.3 \%$

FIGURE 5 | Machine Learning technique distribution.

example, Moreno et al. (9) compared SVM and RF models in binary classification tasks with 2,964 input features for different cardiac pathologies, such as HF or HCM, using optical flow features in cardiac MRI, where the latter model obtained the best performance in most cases. In this case, each DT in the RF model had two depth levels for fast predictions in clinical practice. In another example, Wong et al. (27) a RF outperformed a SVM for infarction detection by means of regional intensity analysis and motion modeling. As a final example, a RF was also used by Baeßler et al. (28) to find the most discriminative features in texture analysis for T1-weighted cardiac MRI for HCM and normal patients classification.

\subsubsection{Cluster Analysis}

Cluster analysis relates to the set of techniques that group together subjects in the form of data points according to similarity or proximity in the parametric space given by quantitative data extracted from input variables (image parameters and/or clinical information), as can be seen in Figure 6D. This technique is very useful for patient stratification, since patients with apparently similar pathology, according to existing image analysis techniques, may fall into previously unrecognized subsets which may inform understanding of disease pathophysiology and inform more effective targeted therapies. Some clustering techniques require definition of outcomes, which means that lay on the unsupervised learning ML group. However, in classification tasks a very common supervised clustering strategy is k-nearest neighbors ( $\mathrm{kNN}$ ) clustering, where $\mathrm{k}$ is the number of neighbor subjects to look at when finding subgroups. In this case, surrounding diagnosed subjects will determine the outcome for a new patient. Most of the reviewed literature in clustering uses $\operatorname{kNN}(29,30)$.

Additional studies report the use of different cluster analysis for classification and/or discovery of cardiac pheno-groups. For example, Bruse et al. (31) used hierarchical clustering techniques to subdivide 60 patients into three groups, a healthy cohort and two associated with congenital heart disease by using shape features from cardiac MRI. Wojnarski et al. (32) also used a cluster analysis technique to group bicuspid aortic valve patients using CT data to find three phenotypes, and a RF was applied later to identify biomarker differences for these phenotypes using echo and clinical data.

\subsubsection{Artificial Neural Network (ANN)}

ANNs are motivated by the structure and interactions of biological neural networks. These models propagate input data in a hierarchical fashion through internal nodes in different layers. Each input line has a corresponding weight that must be estimated and iteratively adjusted during the training process. The ANN adapts until the weights giving optimal model performance are identified (Figure 6E). A nonlinear function is applied in each node to the contribution from incoming connections for obtaining its value/activation (net input function). Weight optimization provides the model with great adaptability to complex boundaries separating classes because of the high non-linear combinations of features involved in such models. Moreover, the connections between layers in an ANN can be used to design different networks depending on the application. Some caveats are the lack of an underlying theory for deciding the amount of layers or nodes in each layer, that depends on each problem and the amount of training data, as well as the trend for these models to adapt to the training set due to the large difference between number of parameters/weights of the model and training samples. ANNs are the best choice when large amount of data is available.

In the literature, these techniques have been applied frequently. For example, Tsai et al. (33) used ANNs for detection of HCM and DCM patients using features extracted from echo. And more recently, two works by Nakajima et al. $(34,35)$, with the same SPECT dataset with 1,001 cases, used ANNs to assess $\mathrm{CAD}$ using features extracted from stress and rest images with good accuracy.

\subsubsection{Convolutional Neural Network (CNN)}

$\mathrm{CNNs}$ are an extension of ANNs in which the value of a node in a given layer is affected by the spatial surrounding 
A

\section{Logistic Regression}

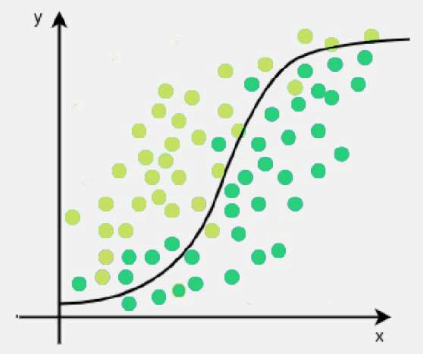

C

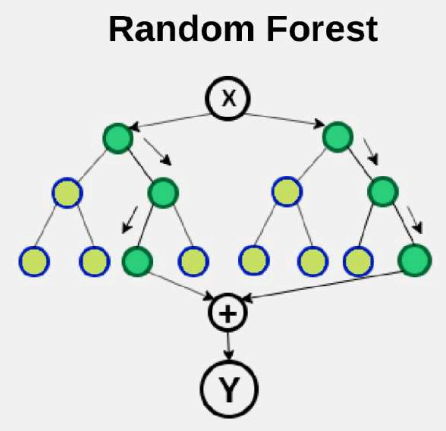

E
B

\section{Support Vector Machine}

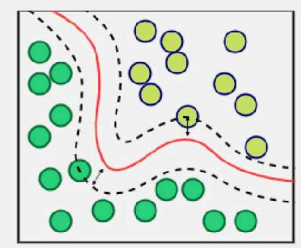

$\theta$

$\theta$
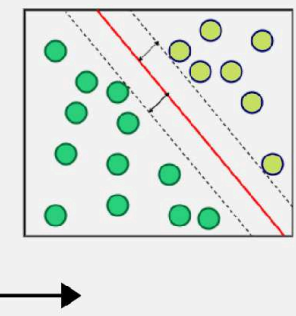

D

\section{Clustering}

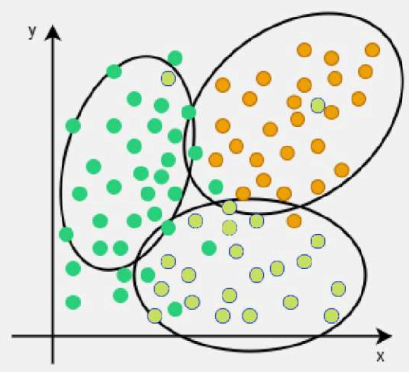

F Convolutional Neural Network

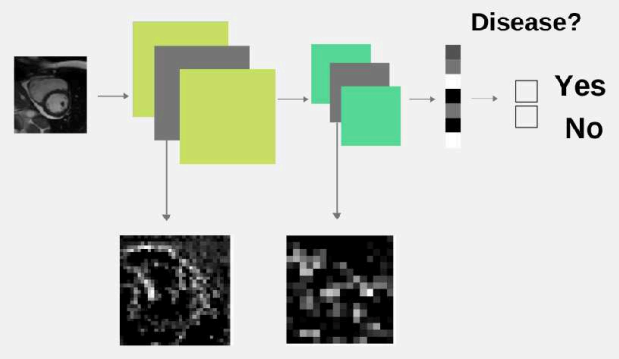

FIGURE 6 | Selected machine learning techniques. (A) Logistic Regression is used to model the probability of a binary outcome. In the figure, $Y$ axis represents the probability while $X$ axis is the continuous input variable. Notice that small changes in $X$ produce large variations of the final probability $Y$, mainly in the central part of the plot where the uncertainty of the model is larger. This model can be extended to a multi-class problems. (B) Support Vector Machine models are able to transform a non-linear boundary to a linear one using the kernel trick. During the training process, the distance between classes to the final selected boundary is maximized. (C) Random Forest is a technique that combines Decision Trees for reducing the uncertainty in the final prediction. It is based in a recursive binary splitting strategy where upper nodes are intended to be the most discriminative ones and subsequent branching is applied to less relevant variables. (D) Clustering is a technique with capability to find subgroups (clusters) along data. There are different cluster techniques, some need a prior number of clusters (kMeans), some of them can be used with output information (KNN), and others are fully unsupervised (meanShift). (E) Artificial neural networks are able to model complex non-linear relations between input variables and outcomes by propagating structured data (green nodes -input variables), e.g., radiomics, through hidden layers (blue nodes) to obtain an output (orange nodes). (F) Convolutional neural networks are the backbone of Deep Learning applications. They comprise input and output layers separated by multiple hidden layers. Their ability to hierarchically propagate imaging information and extract data-driven features implies automatic detection of relevant cardiac imaging biomarkers within the intermediate layers.

of a node in the previous layer through an operation called convolutional product. These models are specially designed for image processing, where spatial information for the nodes (pixels) is essential for the final prediction. The advantages and disadvantages are shared with ANNs. The main difference that make these models very popular nowadays is that images are provided as input without any feature extraction. These models are able to extract their own meaningful features for the final prediction, as illustrated in Figure 6F. Additional models exist for compressing images to a lower dimensional representation space such as the Variational Autoencoder (VAE) and Generative Adversarial Networks (GANs) where additional analysis can be carried out more easily (e.g., clustering or classification with a SVM model). 
TABLE 5 | Common normalization techniques.

\begin{tabular}{lll}
\hline Technique & Description & Advantages \\
\hline $\begin{array}{ll}\text { Mean/variance } \\
\text { normalization }\end{array}$ & $\begin{array}{l}\text { Centering to zero mean } \\
\text { and unit variance }\end{array}$ & $\begin{array}{l}\text { Avoid high variance features } \\
\text { dominance }\end{array}$ \\
Range & Mapping to a given & Robustness to small variances, \\
scaling & interval & preserve zero entries \\
Robust & Mapping interval with & Robustness to outliers \\
scaling & interquartile information & \\
Image & Brightness/contrast & Avoid variability in pixel \\
normalization & correction & intensity distribution
\end{tabular}

A balanced approach should be taken to defining the layers of a CNN; whilst a deeper network loses information from the original image with each new layer, a network with few layers could have problems extracting meaningful features for the final prediction. CNNs are widely used for analysis of images and their application to cardiac imaging is reported in numerous studies. Wolterink et al. (17) presented a framework where two cascading CNNs were able to detect CAC using cardiac computed tomography angiography (CTA) images. Their models had $8-13$ convolutional layers that reduced $200 \times 200$ features (pixel intensities) to only 32. Zhang et al. (36) used a 13-layer CNN to diagnose HCM, cardiac amyloidosis and pulmonary artery hypertension from echo images of size $224 \times 224$, that were reduced to 4,096 features. Madani et al. (37) used a CNN model to predict left ventricular hypertrophy from echo images of size $120 \times 160$.

\subsubsection{Additional Steps}

\subsubsection{Normalization}

Due to the diverse nature of different information sources in cardiac medicine, a normalization step is often required prior to model crafting. In general, learning algorithms benefit from standardization of the data set, e.g., some algorithms as SVM will improve cardiovascular predictions if all numerical features are zero centered and have a variance of the same magnitude order. Furthermore, some non-linear transformations can prepare the selected features to create a model more robust to outliers. Some of the most common techniques are mentioned in Table 5.

For illustration, Wong et al. (27) shows that feature normalization has a positive impact in the ML model performance. Moreover, categorical variables should be encoded using Integer encoding, that consist in referencing each possible categorical value with an integer, or One-Hot encoding, that considers each possible categorical value as a new binary variable.

\subsubsection{Dimensionality reduction and feature selection}

Frequently, after extracting features from different sources such as demographic and clinical data, conventional indices and imaging parameters, one ends up with thousands of values defining a single patient. This information is later utilized during the training process of ML models, but the combination of a large number of input parameters with a limited number of samples (as usually happens in the medical field) can make the optimization problem expensive and may limit the generalization ability of our model. Thus, a dimensionality reduction algorithm is usually applied to the input data, such as principal component analysis (PCA) or linear discriminant analysis (LDA). Another proactive approach is feature selection. Such method will add sequentially the most discriminative features for the particular model instance being trained and dismiss redundant and noninformative ones.

For example, Tabassian et al. (29) aimed to analyze deformation curves of the LV in echocardiographic records of 120 patients. The strain curves obtained were reduced by means of PCA and the result was used to train a strain kNN model. The resultant accuracy was 0.87 , significantly higher than the clinician's results, 0.7. Cetin et al. (38) identified HHD from healthy controls in 200 subjects with SVM and sequential forward feature selection. The predictive power of selected radiomics ( $A U C=0.76$ ) was substantially improved compared to conventional indices (AUC $=0.62$ ).

\subsubsection{Validation}

In order to prove the validity of ML applied to cardiac imaging, results must be analyzed from two perspectives: statistical validity, considering the reproducibility with different cohorts and correctness of statistical values obtained (i.e., metrics), and intra-validity, regarding the clinical and real implications of the algorithms on a daily basis (i.e., clinical effectiveness). This is a pairwise co-existence; none of the ML cardiac imaging algorithms will be applied in clinical routine if there is no agreement from both sides. The following sub-sections will describe how the metrics and the clinical effectiveness are considered.

A cohort is sorted in a very specific manner for ML purposes. For the validity of the algorithms, a whole cardiac imaging data set should be split into 3 different subgroups, called training set, validation set, and testing set, respectively. These groups are often selected in such way that subgroups share demographic distributions such as age or sex, in order to represent a real world scenario. Of course, a balanced distribution of control and pathologic subjects is also required. Once the ML model is trained and tested, different metrics are obtained to evaluate its performance.

Accuracy measures the percentage of the algorithm classifying the input data correctly. It is a simple measure used in multiple scientific scenarios if there is no class imbalance (i.e., one class represented by a higher number of individuals compared with the rest). One of the drawbacks of using accuracy as the metric is that there is a knowledge loss when measuring False Positive and False Negative observations. Therefore, Specificity (Sp) and Sensitivity (Se) are widely used for measuring the performance of the algorithm, this time taking into consideration a possible class imbalance. In order to assess the performance of an algorithm and to understand where there might be a miss-classification issue, a table report called Confusion Matrix is used. This specific table layout is typically used to describe the performance of a supervised learning model. Each row of the matrix represents the instances in a predicted class while each column represents the instances in an actual class (or vice versa). This way, a computer scientist can have a wider 

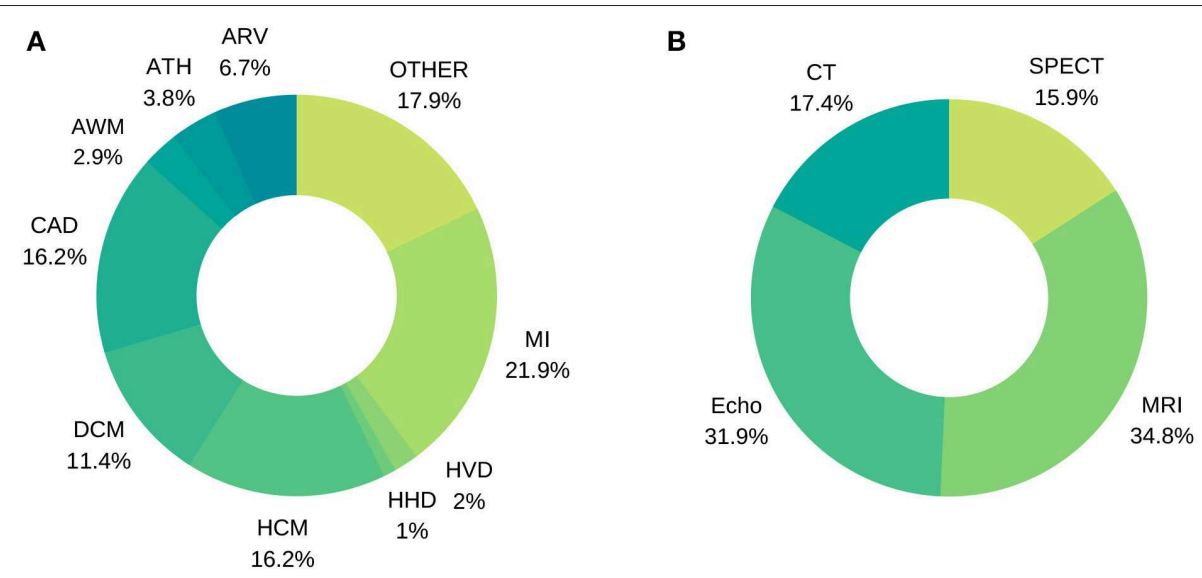

FIGURE 7 | Distribution of image-based diagnostic application using machine learning (A) per disease, (B) per modality.

overview of the parameters that may be changed or which classes are down-performing the algorithm. From sensitivity, specificity and the confusion matrix we can extract a performance plot representation called the receiver operating (ROC) curve. It is created by plotting the true positive rate (TP rate) against the false positive rate (FP rate) at various threshold settings. In ML, the true-positive rate is also known as sensitivity, recall or probability of detection. ROC analysis is related in a direct and natural way to cost/benefit analysis of diagnostic decision making. The area under the ROC curve (AUC) is another metric used to measure algorithms' performance.

It is noticeable that AUC can be derived from decision boundaries obtained by ML models despite the fact that it is trained with discrete outputs. When a trained model is asked to make a prediction, a probability can be computed and used to generate a ROC analysis.

\section{DIAGNOSTIC APPLICATIONS-A REVIEW OF LITERATURE}

We conducted an organized, pre-defined literature search of two electronic databases (Google Scholar, Scopus). We included studies using a well-defined ML technique for cardiac image analysis using echocardiography, cardiac magnetic resonance, cardiac computed tomography, or single photon emission computed tomography (SPECT). Our search strategy comprised a series of title and whole text searches with search terms combined using Boolean operators. Search results were filtered by subject area, limiting to entries from Cardiology, Computer Science and Engineering fields. We review in detail various achievements in the diagnosis of a wide range of cardiac diseases using image-based ML methods. Statistics about the conducted literature review can be seen in Figure 7.

\subsection{Myocardial Infarction}

Accurate and timely identification of MI helps in guidance of treatment strategies and reduction in the time taken for further tests. While MI diagnostic assessment using imaging is prone to inter- and intra-observer variability and requires significant amount of time of experts, ML methods offer opportunities to simplify, speed up and quantify the diagnostic process in combination with conventional assessment. For example, Nakada et al. (39) demonstrated that MI diagnosis can be achieved in echo using quantitative motion features, avoiding the interobserver human variability, as input for an ANN reaching an accuracy of 0.95. Later, Ungru et al. (40) validated these results in mice models by inducing MI in healthy specimens with a prediction accuracy of 0.91 , comparing several ML techniques. The same level of accuracy was obtained in the first texture analysis work, by Agani et al. (41), with only 17 subjects and a clustering approach. This echocardiographic research was later extended with a full pool of texture features and 160 subjects by Sudarshan et al. (42). In this work, DT, ANN and SVM models were benchmarked, with the best accuracy obtained using ANN: 0.94 ( $\mathrm{Se}=0.91, \mathrm{Sp}=0.97$ ). Vidya et al. (43) also performed an intensive texture analysis for 800 subjects, achieving an accuracy of 0.99 using a SVM. In their study, different pre-processing techniques were used to enhance the cardiac images.

Cardiac MRI has particular value in identification of MI. Since 2017, 13 studies were found integrating input variables from this imaging modality. Baeßler et al. (44) used late gadolinium enhancement MRI as a standard reference for non-enhanced MRI discrimination between chronic and subacute MI. Radiomic features in combination with a LR gave an AUC of 0.92 in a cohort of 180 patients. Similarly, segment viability can be detected on cine MRI using also radiomics, as suggested by Larroza et al. (45). This classification between viable, nonviable and remote segments yielded an AUC of 0.84. However, we believe that these encouraging results should be validated with a bigger cohort, and a well-balanced segment viability distribution. Recently, Zhang et al. (46) tried to detect MI from non-enhanced MRI images. 212 patients with chronic MI and 87 healthy control patients were used to train a three-stage DL pipeline. The per-segment AUC for detecting chronic MI was $0.94(\mathrm{Sp}=0.99, \mathrm{Se}=0.9)$

Two consecutive state-of-the-art texture analysis studies were conducted in cardiac CT: Mannil et al. (47) and Mannil et al. 
TABLE 6 | Selected studies using image-based ML analysis for the diagnosis of Myocardial Infarction.

\begin{tabular}{|c|c|c|c|c|c|c|}
\hline Publication & Modality & Biomarker & ML technique & Diagnostic & Sample size & Performance \\
\hline (44) & $\mathrm{MRI}$ & Radiomics & LR & $\mathrm{Ml}$ & 180 & $\mathrm{ACC}=0.92$ \\
\hline (46) & $\mathrm{MRI}$ & Conventional & ANN & $\mathrm{Ml}$ & 299 & $A \cup C=0.94$ \\
\hline (45) & $\mathrm{MRI}$ & Radiomics & SVM & $\mathrm{Ml}$ & 50 & $A \cup C=0.84$ \\
\hline (9) & $\mathrm{MRI}$ & Conventional & SVM/RF & $\mathrm{Ml} / \mathrm{HCM}$ & 45 & $\mathrm{ACC}=0.94$ \\
\hline (94) & $\mathrm{MRI}$ & Conventional & DT/CL/SVM & $\mathrm{Ml}$ & 200 & $\mathrm{ACC}=0.95$ \\
\hline (95) & $\mathrm{MRI}$ & Conventional & PLS & $\mathrm{Ml}$ & 200 & $A C C=0.98$ \\
\hline (22) & Echo & Qualitative & SVM & $\mathrm{Ml}$ & 242 & $A C C=0.97$ \\
\hline (39) & Echo & Conventional & ANN & Ml/AP & 91 & $\mathrm{ACC}=0.95$ \\
\hline (40) & Echo & Conventional & BN/DT/CL/SVM & $\mathrm{Ml}$ & 42 & $\mathrm{ACC}=0.91$ \\
\hline (42) & Echo & Radiomics & DT/ANN/SVM & $\mathrm{Ml}$ & 160 & $\mathrm{ACC}=0.94$ \\
\hline (41) & Echo & Radiomics & $\mathrm{CL}$ & $\mathrm{Ml}$ & 17 & $\mathrm{ACC}=0.91$ \\
\hline (43) & Echo & Radiomics & SVM & $\mathrm{Ml}$ & 800 & $\mathrm{ACC}=0.99$ \\
\hline (29) & Echo & Conventional & $\mathrm{CL}$ & $\mathrm{Ml}$ & 120 & $\mathrm{ACC}=0.87$ \\
\hline$(47)$ & $\mathrm{CT}$ & Radiomics & RF/CL/ANN & $\mathrm{Ml}$ & 87 & $A C C=0.78$ \\
\hline (48) & CT & Radiomics & DT & $\mathrm{Ml}$ & 30 & $A C C=0.97$ \\
\hline$(27)$ & CT & Conventional & SVM/RF & $\mathrm{Ml}$ & 170 & $A C C=0.85$ \\
\hline (96) & SPECT & Conventional & $\mathrm{BN}$ & Ml/CAD & 728 & $\mathrm{ACC}=0.78$ \\
\hline
\end{tabular}

(48). The former underlines ML ability for detecting MI on noncontrast low radiation dose $\mathrm{CT}$ images on the basis of features invisible to the radiologists' eye, obtaining an AUC of 0.78 . The latter study evaluates the impact of automatic classification methods using different iterative reconstruction (IR) strengths for contrast-enhancement images, reporting an accuracy of 0.94 (IR 3) and 0.97 (IR 5) for the ML model, while three independent readers achieved 0.73 (IR 5) on average. A summary of MI studies can be found in Table 6 .

\subsection{Cardiomyopathies}

Cardiomyopathy is a broad term describing various heart muscle disorders, a first level of subclassification is into ischaemic and non-ischaemic cardiomyopathies. This heterogenous group of disorders have many causes, signs and symptoms, and require different treatments. The challenge of distinguishing different cardiomyopathies is illustrated by the fact that many of them can be associated with diverse manifestations. Each disease entity is associated with a typical imaging phenotypes. Whilst in routine image analysis, it is not always possible to discriminate individual cardiomyopathies, this may be improved with the more granular and quantitative approach to image analysis in ML models. These premises makes ML-based imaging diagnosis a perfect tool for computer aided analysis of heterogeneous cardiomyopathies. For example, Gopalakrishnan et al. (49) used a set of conventional indices from a pediatric cardiac MRI cohort of 83 subjects to characterize five different cardiomyopathies. In this study, a DT $($ AUC $=0.79)$ was compared with other ML methods (AUC $=0.73-0.77)$. Physiological vs. pathological patterns of HCM remodeling were characterized by Narula et al. (50) using an ensemble of models with conventional indices from $2 \mathrm{D}$ echo as input $(\mathrm{Se}=0.96, \mathrm{Sp}=0.77)$.

In 2017, a relevant challenge was organized by Bernard et al. (51). The Automated Cardiac Diagnosis Challenge (ACDC) aimed to evaluate the performance of different automatic methods for the classification of 150 subjects into 5 categories (healthy, HCM, DCM, ARV and MI) as provided by clinical experts. Several approaches were proposed for this problem. Khened et al. (4) and Wolterink et al. (52) used a set of conventional indices extracted from their own automatic delineations as input for a RF to obtain an accuracy of 0.96 and 0.86 on the test set, respectively. Isensee et al. (53) also used a $\mathrm{RF}$ and their own segmentation scheme to classify cardiac cycle dynamic features, with an accuracy of 0.92 . From this study, the benefit of the addition of temporal analysis is remarkable and provides a strong argument to be exploited further in future cine MRI studies. Cetin et al. (14) used SVM to classify a complete pool of radiomic features from manual segmentation, obtaining also an accuracy of 0.92. Additional research has been done later using the same dataset. Snaauw et al. (54) proposed a novel approach, using CNN bottleneck representations to discriminate between the five categories, obtaining a modest accuracy of 0.78 . Another interesting approach was taken by Biffi et al. (55). Their VAE architecture was trained with two multi-center cohorts of 537 and 200 patients and tested on their own dataset and on the ACDC dataset, obtaining an accuracy of 1.0 and 0.9 , respectively.

Later, Puyol-Antón et al. (56) combined MRI and echo data and per-segment motion analysis to diagnose DCM by means of LDA, achieving an accuracy of 0.94 ( $\mathrm{Sp}=0.96$, Se $=0.93$ ). Recently, Neisius et al. presented two complementary works approaching HCM and HHD diagnosis from two different perspectives, Neisius et al. $(15,57)$. In the first work, a complete strain analysis and a LR achieved an accuracy of 0.67 ( $\mathrm{Sp}=0.64$, $\mathrm{Se}=0.68$ ). The second one applied an exhaustive texture analysis for T1 mapping. A selection of 6 radiomic texture features and a linear SVM model showed an improved accuracy of 0.86 (Sp = 0.91 , Se $=0.77$ ). A summary of cardiomyopathy studies can be found in Table 7. 
TABLE 7 | Selected studies using image-based ML analysis for diagnosis of various cardiomyopathies.

\begin{tabular}{|c|c|c|c|c|c|c|}
\hline Publication & Modality & Biomarker & ML technique & Diagnostic & Sample size & Performance \\
\hline (49) & $\mathrm{MRI}$ & Conventional & $\mathrm{BN}$ & HCM/DCM/ARV/MYO & 83 & $A \cup C=0.79$ \\
\hline (28) & $\mathrm{MRI}$ & Radiomics & RF/LR & $\mathrm{HCM}$ & 62 & $A \cup C=0.95$ \\
\hline (52) & $\mathrm{MRI}$ & Conventional & $\mathrm{RF}$ & Ml/HCM/DCM/ARV & 100 & $A C C=0.86$ \\
\hline (14) & $\mathrm{MRI}$ & Radiomics & SVM & Ml/HCM/DCM/ARV & 100 & $\mathrm{ACC}=0.92$ \\
\hline (53) & $\mathrm{MRI}$ & Conventional & RF & Ml/HCM/DCM/ARV & 100 & $\mathrm{ACC}=0.92$ \\
\hline (4) & $\mathrm{MRI}$ & Conventional & RF & Ml/HCM/DCM/ARV & 100 & $A C C=0.96$ \\
\hline (55) & $\mathrm{MRI}$ & Deep Learning & VAE & $\mathrm{HCM}$ & 737 & $A C C=1.00$ \\
\hline (10) & MRI & Conventional & LR & Ml/HCM/DCM/ARV & 100 & $\mathrm{ACC}=0.94$ \\
\hline (57) & $\mathrm{MRI}$ & Conventional & LR & $\mathrm{HHD} / \mathrm{HCM}$ & 224 & $\mathrm{ACC}=0.67$ \\
\hline (15) & $\mathrm{MRI}$ & Radiomics & SVM & $\mathrm{HHD} / \mathrm{HCM}$ & 224 & $A C C=0.86$ \\
\hline (54) & $\mathrm{MRI}$ & Deep Learning & CNN & Ml/HCM/DCM/ARV & 100 & $A C C=0.78$ \\
\hline (9) & $\mathrm{MRI}$ & Conventional & SVM/RF & $\mathrm{Ml} / \mathrm{HCM}$ & 45 & $\mathrm{ACC}=0.94$ \\
\hline (31) & $\mathrm{MRI}$ & Conventional & $\mathrm{CL}$ & $\mathrm{CHD}$ & 60 & $\mathrm{ACC}=0.89$ \\
\hline (50) & Echo & Conventional & SVM/RF/ANN & $\mathrm{HCM} / \mathrm{ATHCM}$ & 139 & $A C C=0.91$ \\
\hline (33) & Echo & Radiomics & ANN/GA & HCM/DCM & 90 & $A C C=0.95$ \\
\hline (18) & Echo & Deep Learning & CNN & HCM/DCM & 927 & $A \cup C=0.84$ \\
\hline (56) & Echo/MRI & Conventional & SVM & DCM & 69 & $\mathrm{ACC}=0.94$ \\
\hline (26) & Echo & Radiomics & SVM & DCM/ASD & 439 & $\mathrm{ACC}=0.98$ \\
\hline (37) & Echo & Deep Learning & CNN/GAN & $\mathrm{HCM}$ & 772 & $A C C=0.92$ \\
\hline (36) & Echo & Deep Learning & CNN & $\mathrm{HCM} / \mathrm{CA} / \mathrm{PH}$ & 14,035 & $A \cup C=0.93$ \\
\hline
\end{tabular}

\subsection{Coronary Artery Disease}

Non-invasive imaging assessment for detection of CAD has a great potential impact on clinical practice. If ischemia can be discarded with a high probability, invasive coronary angiography (ICA) may be avoided. Advanced ML image analysis techniques can improve the diagnostic accuracy of myocardial ischemia and through this improve CAD management and reduce unnecessary downstream testing.

A very first approach dating from 1999 showed promising results. Considering ICA as reference standard, Kukar et al. (58) used scintigraphy, ECG and data on symptoms from 327 patients to detect CAD. Different ML models and feature selections were tested and in some cases the ML model outperformed clinicians in accuracy ( 0.92 vs. 0.91 , respectively), but not in sensitivity. An exhaustive approach by Kurgan et al. (59) sets the base for a semi-automated diagnosis pipeline in perfusion SPECT. In their work, a pseudo-DT was crafted from intensitybased features, for 267 subjects, achieving an overall accuracy of 0.8 . Another similar work was conducted in perfusion SPECT ( $n=115)$ and Equilibrium Radionuclide Angiocardiography ( $n=58$ ) by Bagher-Ebadian et al. (8). Using ICA as ground truth for both studies, CAD was assessed using mean and variance intensity features extracted from stress and rest studies in anterior, left anterior oblique and left lateral projections, obtaining accuracies of 0.77 and 0.73 with an ANN. A similar methodology was covered in detail by Guner et al. (60). A cohort of 308 patients with clinical coronary CTA assessment was utilized to train an ensemble of ANNs for CAD discrimination. A combination of demographic information and frequency, phase and brightness features provided as input variables resulted in model accuracy of 0.74 , outperforming some of the non-expert clinicians. The results revealed that single-vessel CAD was more difficult to identify. Recently, complementary work by Shibutani et al. (61), including per-segment analysis, was performed on 21 patients who underwent perfusion SPECT. A total of 109 abnormal regions were examined and an ANN achieved better results than two independent observers for stress defect and ischemia detection, with respect to ICA as gold standard.

Alternatively, resting CT can be used for CAD diagnosis without additional contrast injection for stress imaging. Han et al. (62) used 3 quantitative features and the 17-segment model to obtain 51 input variables for training a gradient boosting algorithm, a ML technique that builds an ensemble of classifiers to improve the final accuracy. Invasive angiography and FFR were used as gold standard. This study based on a 252 patients' cohort from 5 countries and 17 centers, obtained an AUC of 0.75. Another state-of-the-art approach using cardiac CT, by Coenen et al. (63), showed that improved reclassification of nonsignificant stenosis is possible with ML-based image analysis. Three hundred and fifty-one patients, including 525 vessels with invasive FFR comparison were included in this study. A set of 28 anatomical features were computed from semi-automatic 3D CT reconstructions. On a per-vessel basis, diagnostic accuracy improved from 0.58 (CTA) to 0.78 (ML model). The per-patient accuracy improved from 0.71 to 0.85 . A summary of CAD studies can be found in Table 8 .

\subsection{Atherosclerosis}

Atherosclerosis is a strong and independent predictor of cardiovascular events. Plaque is often scored manually by experts, which leads to an increase in workload, is prone to 
TABLE 8 | Selected studies using image-based ML analysis for diagnosis of coronary artery disease.

\begin{tabular}{|c|c|c|c|c|c|c|}
\hline Publication & Modality & Biomarker & ML technique & Diagnostic & Sample size & Performance \\
\hline (20) & SPECT & Conventional & LB & CAD & 1,181 & $A \cup C=0.94$ \\
\hline (16) & SPECT & Deep Learning & CNN & CAD & 1,160 & $A \cup C=0.81$ \\
\hline (34) & SPECT & Conventional & ANN & CAD & 1,365 & $A \cup C=0.75$ \\
\hline (35) & SPECT & Conventional & ANN & CAD & 106 & $A \cup C=0.96$ \\
\hline (60) & SPECT & Conventional & ANN & CAD & 65 & $A \cup C=0.74$ \\
\hline (97) & SPECT & Conventional & DT/GA & CAD & 267 & $A C C=0.83$ \\
\hline (24) & SPECT & Qualitative & SVM & CAD & 267 & $\mathrm{ACC}=0.92$ \\
\hline (8) & SPECT & Deep Learning & ANN/CL & CAD & 173 & $A \cup C=0.80$ \\
\hline (61) & SPECT & Conventional & ANN & CAD & 109 & $A \cup C=0.88$ \\
\hline (6) & PET & Conventional & N/A & CAD/MACE & 1,234 & $A \cup C=0.72$ \\
\hline (63) & CT & Conventional & $\mathrm{N} / \mathrm{A}$ & CAD & 352 & $A \cup C=0.84$ \\
\hline (62) & CT & Conventional & GBRT & CAD & 252 & $A \cup C=0.75$ \\
\hline (58) & echo/SCl & Qualitative & ANN & CAD & 327 & $\mathrm{ACC}=0.80$ \\
\hline (98) & echo & Radiomics & SVM & CAD & 61 & $A \cup C=0.88$ \\
\hline (25) & echo & Qualitative & SVM & CAD & 228 & $\mathrm{ACC}=0.99$ \\
\hline
\end{tabular}

false positives and to inter-observer variability regarding CAC detection. Hence, the ability to quickly and reliably quantify calcification using ML models provides additive value to clinical risk scoring tools and will enable superior prognostication of individuals. To overcome these issues and bring robustness to such procedures, intensive cardiac imaging feature extraction may be utilized.

Išgum et al. (30) designed an automated method for detection of aortic calcification, an indicator of established atherosclerotic disease, based on shape and intensity features. Forty abdominal scans contained a total of 249 CAC determined by a human observer. The method detected $209 \mathrm{CAC}(\mathrm{Se}=0.84)$ at the expense of 1.0 false-positive object per scan on average, while the presence of contrast increased the number of incorrect classifications. This work was complemented by Išgum et al. (64), analysing cardiac CT with a more sophisticated feature set to obtain a final accuracy of 0.74 for CAC detection. Feature selection showed that no shape features were included in the classification stage, highlighting the discriminating power of texture analysis in CT.

Wolterink et al. (65) used cardiac CT scans thresholded at 130 Hounsfield units and a connected-component analysis to obtain candidate regions in the coronary arteries for 164 subjects with expert annotations. Their texture analysis was similar to Išgum et al. (64), and the resulting accuracy with DTs was 0.86 for risk stratification. This work also introduced a guided review where the most uncertain CAC were manually inspected again, increasing the overall accuracy up to 0.92 . Later, a large radiomic pool of 4,440 features was extracted from a group of 60 subjects with Napkin Ring Sign (NRS) and nonNRS plaques with similar degree of manually segmented CAC by Kolossváry et al. (66). This research unveils the value of radiomics to find discriminative features: almost half of them reached an AUC of 0.8, short- and long-run low gray-level emphasis and surface ratio of high attenuation voxels had the highest AUC values (0.92 and 0.89, respectively). Finally, in a recent work, Zreik et al. (67) used recurrent CNNs in multiplanar reformatted coronary CTA images previously annotated by an expert, achieving accuracies of 0.77 and 0.8 for plaque and stenosis characterization, respectively. A summary of ATH studies can be found in Table 9.

\subsection{Valvular Heart Disease}

Heart valve disease is an increasingly common pathology of the cardiovascular system and an increasing number of patients are expected to require heart valve replacement. Such diverse group of disorders can benefit from cardiac imaging ML integration through early diagnosis, treatment or surgery planning. For instance, Elalfi et al. (68) used imaging preprocessing techniques (Gaussian and Gabor filtering) and intensity and texture features to generate an ANN model with 120 echo images. These images were organized in 8 types of valvular diseases. The obtained accuracy was high at 0.93 . This is encouraging particularly considering the diversity of outcomes.

A similar approach was addressed for mitral regurgitation (MR) severity estimation using echo videos. Moghaddasi et al. (69) took advantage of binary patters as image descriptors which include details from different viewpoints of the heart. $\mathrm{kNN}$ and SVM models were trained with 102 patients divided in four groups: mild $\mathrm{MR}(n=34)$, moderate $\mathrm{MR}(n=32)$, severe MR $(n=36)$, and control $(n=37)$. SVM obtained the best accuracy, 0.99 . Another interesting work mentioned in previous sections was conducted by Wojnarski et al. (32). A summary of HVD studies can be found in Table $\mathbf{1 0 .}$

\subsection{Heart Failure}

Heart failure with preserved ejection fraction (HFpEF) is a heterogeneous group of disorders with variable treatment response and poor outcomes. There has been increasing interest in improved phenotyping of HFpEF to aid understanding of underlying disease mechanisms and also to guide treatments toward subtypes who may derive benefit. Given 
TABLE 9 | Selected studies using image-based ML analysis for diagnosis of aortic and coronary atherosclerosis.

\begin{tabular}{|c|c|c|c|c|c|c|}
\hline Publication & Modality & Biomarker & ML technique & Diagnostic & Sample size & Performance \\
\hline (66) & CT & Radiomics & N/A & ATH & 60 & $A \cup C=0.91$ \\
\hline (67) & $\mathrm{CT}$ & Deep Learning & CNN & ATH & 163 & $A C C=0.80$ \\
\hline (65) & CT & Radiomics & DT & ATH & 164 & $A C C=0.86$ \\
\hline (17) & CT & Deep Learning & CNN & ATH & 250 & $A C C=0.72$ \\
\hline (64) & CT & Conventional & $\mathrm{CL}$ & ATH & 615 & $\mathrm{ACC}=0.74$ \\
\hline (30) & CT & Conventional & $\mathrm{CL}$ & ATH & 249 & $A C C=0.83$ \\
\hline
\end{tabular}

TABLE 10 | Selected studies using image-based ML analysis for diagnosis of valvular heart disease.

\begin{tabular}{|c|c|c|c|c|c|c|}
\hline Publication & Modality & Biomarker & ML technique & Diagnostic & Sample size & Performance \\
\hline (68) & echo & Radiomics & ANN & HVD & 120 & $\mathrm{ACC}=0.93$ \\
\hline (69) & echo & Radiomics & SVM/CL & HVD & 102 & $\mathrm{ACC}=0.99$ \\
\hline (32) & CT & Conventional & $\mathrm{CL}$ & HVD & 656 & $\mathrm{~N} / \mathrm{A}$ \\
\hline
\end{tabular}

TABLE 11 | Selected studies using image-based ML analysis for diagnosis of heart failure.

\begin{tabular}{|c|c|c|c|c|c|c|}
\hline Publication & Modality & Biomarker & ML technique & Diagnostic & Sample size & Performance \\
\hline (9) & $\mathrm{MRI}$ & Conventional & SVM/RF & $\mathrm{Ml} / \mathrm{HCM} / \mathrm{HF}$ & 45 & $A C C=0.77$ \\
\hline$(21)$ & $\mathrm{MRI}$ & Radiomics & LR & $\mathrm{HF}$ & 79 & $A \cup C=0.85$ \\
\hline$(70)$ & echo & Conventional & $\mathrm{CL}$ & HHD/HFePF & 100 & $A C C=0.81$ \\
\hline$(71)$ & echo & Conventional & CL/SVM & HFePF & 397 & $A \cup C=0.76$ \\
\hline (72) & echo & Conventional & $\mathrm{CL}$ & $\mathrm{HF}$ & 1,106 & N/A \\
\hline
\end{tabular}

the heterogeneous nature of HFpEF, ML techniques are a very suitable tool for diagnosis and image phenotype stratification. Some of the reviewed studies in previous sections were also related to the characterization of heart failure $(9,70)$. Additional work in this field was presented by Shah et al. (71), that prospectively studied $397 \mathrm{HFpEF}$ patients and performed detailed clinical, laboratory, electrocardiographic and echocardiographic phenotyping of the study participants. Clustering techniques were applied to divide the cohort into 3 pheno-groups. Phenomapping was helpful for improved classification and categorization of HFpEF patients and risk stratification by means of SVM, obtaining an AUC of 0.76. ML applied to HF phenogrouping is also used for prognostic tasks by Cikes et al. (72). A summary of HF studies can be found in Table 11.

\subsection{Abnormal Wall Motion}

Most of the existing quantitative techniques for wall motion characterization involve laborious post-processing and image analysis. For this reason, ML approaches with a minimum user input and a correlation with the segmental cardiac function can improve clinical routine and triage.

For instance, Mantilla et al. (7) detected wall motion abnormalities in the left ventricle by means of spatiotemporal profiles obtained with pseudo delineations of 20 MRI patients. Wavelet and Fourier transforms were applied and the subsequent spaces were used to generate two models: SVM and dictionary learning (DICTL). Dictionary Learning at mid-cavity level obtained the best accuracy, $0.96(\mathrm{Sp}=\mathrm{Se}=0.96)$. Afshin et al. (73) exploited intensity distributions per segment. In their work, a reference frame automatically propagated to each cardiac phase generated the 16 segments for the whole cardiac cycle. LDA reduced feature dimensionality and linear SVM obtained an accuracy of 0.86 in a cohort of 58 MRI subjects.

Kusunose et al. (19) used a total of 300 patients with a history of myocardial infarction and 100 age-matched control patients. Each case contained echo from short-axis views at end-diastolic, mid-systolic, and end-systolic phases. An ensemble of $10 \mathrm{CNN}$ models were trained. AUC obtained by the ML ensemble was similar to that produced by the cardiologists and sonographer readers ( 0.99 vs. 0.98 , respectively), and the same occurred for territory detection ( 0.97 vs. 0.95 , respectively). A summary of AWM studies can be found in Table 12.

\section{DISCUSSION AND FUTURE PERSPECTIVES}

Reflected by the large amount of already published data reviewed above, AI in general and ML in particular have been shown to exhibit a huge potential to significantly influence diagnostic decision making in cardiology. In contrast to "traditional" statistical methods, the techniques from the field of AI are able to deal with large amounts of data ("big data") and to 
TABLE 12 | Selected studies using image-based ML analysis for diagnosis of wall motion abnormalities.

\begin{tabular}{|c|c|c|c|c|c|c|}
\hline Publication & Modality & Biomarker & ML technique & Diagnostic & Sample size & Performance \\
\hline (7) & $\mathrm{MRI}$ & Conventional & SVM/DICTL & AWM & 20 & $\mathrm{ACC}=0.96$ \\
\hline (73) & $\mathrm{MRI}$ & Conventional & SVM & AWM & 58 & $A C C=0.86$ \\
\hline (19) & echo & Deep Learning & CNN & AWM & 400 & $A \cup C=0.99$ \\
\hline
\end{tabular}

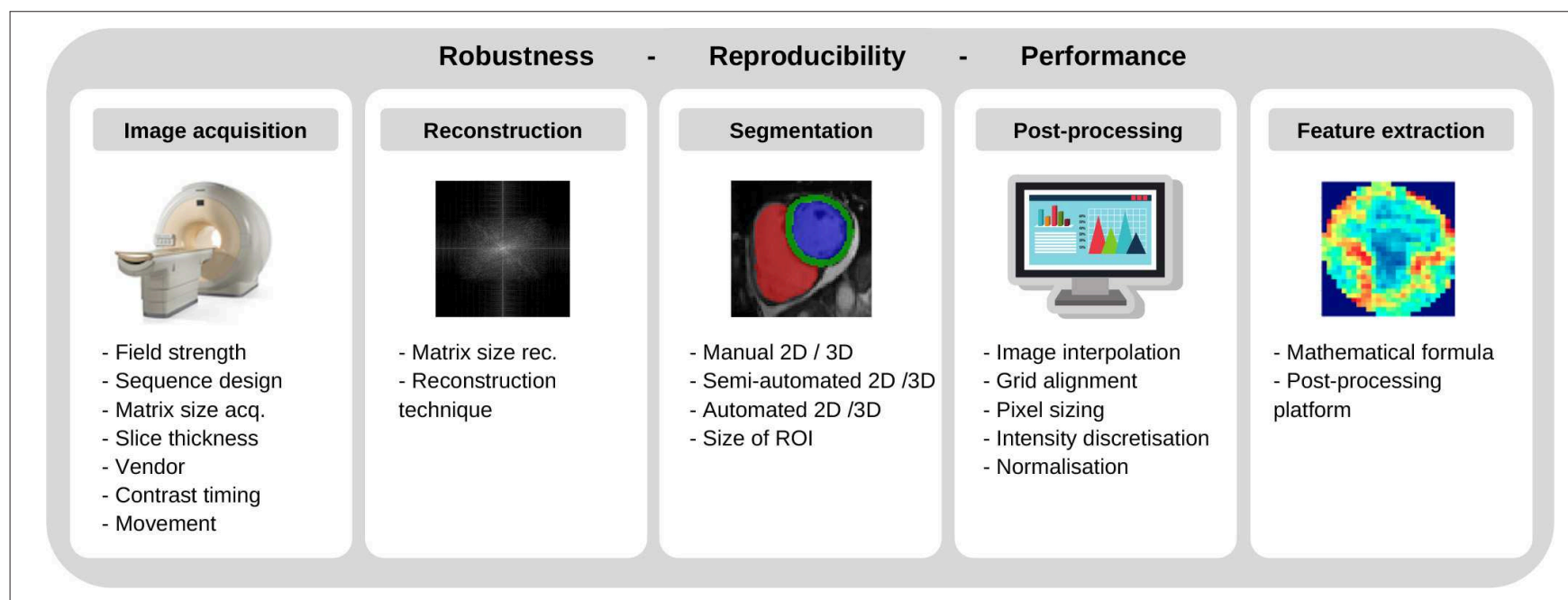

FIGURE 8 | Factors involving robustness and reproducibility of quantitative imaging features.

integrate information from all fields of clinical care, including e.g., clinical parameters ("clinomics"), genetic information ("genomics"), protein metabolism ("proteomics"), and imaging data ("radiomics") within one large all-encompassing analysis framework. The steadily increasing computational power and the increasing availability of data through mobile applications and the digital transformation of the global healthcare systems further contribute to the advancement of the field. Consequently, future studies will continue the use of these techniques in order to allow translation into routine clinical practice and thus pave the way toward improved diagnostic decision making tailored to individual patient-specific needs (subsumed under the heading "precision medicine").

Yet, in today's clinical routine, diagnostic decisions are still drawn from stand-alone parameters [e.g., LV ejection fraction, (74)], despite many encouraging research studies from the field of AI. On a per-patient basis, the diagnostic and prognostic value of such independent functional parameters was found to be low, Park and Kim (75). Given the diversity of cardiovascular imaging modalities, their potential additive value for more accurate diagnostics and risk stratification remains unclear. Besides, continued reliance on subjective visual interpretation, has resulted in considerable observerdependencies and lack of standardization. The application of AI and precision medicine to CVD, however, is currently still is in its infancy, and faces huge challenges which have to be overcome by future research. To establish novel imaging biomarkers and AI techniques, the robustness and reproducibility of quantitative imaging features must be ensured, Zwanenburg et al. (76).
Up to now, trained models and algorithms have limited generalizability due to the multiplicity of potential influencing factors (including differing scanners, vendors, CT radiation doses, MRI field strengths, sequences, sequence parameters, spatial and temporal resolutions, reconstruction algorithms, reconstruction parameters, and so forth; Figure 8).

For CT and positron emission tomography (PET) imaging, a variety of studies have highlighted difficulties in producing reliably reproducible radiomic features when using different vendors, scanners, and acquisition or reconstruction settings (48, 77-84). While the "image biomarker standardization initiative" (IBSI) has established certain standards for radiomic studies, Zwanenburg et al. (76), the specific needs of cardiac imaging have not yet been met. For cardiac CT, Hinzpeter et al. and Mannil et al. have investigated the influence of slice thickness, Hinzpeter et al. (84), and iterative reconstruction algorithms, Mannil et al. (48), on the robustness and comparability of radiomics features - observing considerable feature variations for differing technical settings. In contrast to this evolving body of literature on CT imaging, little evidence exists concerning the robustness of radiomic features in MRI $(75,85-87)$. Given the qualitative nature of most MRI sequences and the absence of absolute signal intensities (in contrast to CT imaging for instance), the robustness of radiomic features seems to heavily depend on acquisition sequences as well as acquisition and reconstruction parameters. In a recent phantom study, Baeßler et al. sought to evaluate the influence of different acquisition sequences, spatial resolution, and postprocessing settings (88) revealing that the robustness of radiomic features was heavily 
influenced by the acquisition sequence and image resolution as well as image processing settings. Future work not only needs to add to the understanding of such influencing factors but should also merge into extensive standardization efforts to ensure reliability of all imaging measures.

Several attempts to improve radiomic feature robustness through image normalization have been made. For more reliable quantification of emphysema, normalization was proposed for chest CT images reconstructed with different kernels, GallardoEstrella et al. (89). The proposed method decomposed each scan into multiple frequency bands, the energy of which was then normalized to the average energies observed in a set of scans reconstructed with a reference kernel. Building on these results, Jin et al. used a deep learning-based strategy for CT image normalization by means of a U-Net, Jin et al. (90). For harmonization of MRI images, similar deep learning algorithms were proposed for dynamic contrast enhanced (DCE) images in breast, Samala et al. (91), and brain MRI, Dewey et al. (92). Although yielding promising results, the applicability of such approaches in cardiovascular applications remains elusive, which is due to inherent particularities of cardiac imaging. Other than breast and brain, the human heart is steadily moving because of breathing and myocardial contraction. Second, the contrast bolus inside the ventricular lumen may influence the myocardial features. Aside from these specific characteristics, the impact of image normalization on extracted radiomic features has not been fully investigated yet. Besides lack of standardization of technical factors, the recent trend to train ML classifiers on relatively small datasets is a major issue of current methodology and hampers translation of the novel techniques into routine clinical practice. The small sample sizes in most cardiovascular imaging studies (usually $\mathrm{N}<100$ with $>1,000$ variables in the models) lead to a considerable risk of overfitting. Overfitting leads to poor generalisability of the classification models when deployed to different datasets. Besides the current lack of imaging feature standardization and the problem of model-overfitting, other challenges should be acknowledged when it comes to translation of AI to daily patient care. While big data aims to integrate data from various sources, the current lack of interoperability of many systems used in clinical care poses huge obstacles for data pooling approaches. Several national and international attempts are currently under way to solve interoperability issues for medical care and to allow a seamless integration of different databases and informatic systems used in healthcare.

The ability to understand the rationale behind ML generated diagnostic grouping may be crucial in order to achieve widespread clinical use of this novel technology. However, especially with DL techniques, those are usually considered as being "black boxes", which do not deliver any insights or explanations on how they reached their conclusions and upon which, e.g., imaging features, they based their decision. Although several attempts and ongoing research exist on delivering insights into an algorithm's decision making (such as heatmaps), these attempts are not sufficiently elaborated so far to convince most cardiology practitioners to use a diagnostic black box in daily clinical patient management.
Thus, interpretability of DL models including the psychological aspects of digital transformation itself should represent one major aim of future research. Radiomics might represent a valid alternative for the meantime, since radiomic modelsin cases where an appropriate and stepwise feature reduction is performed before training the $\mathrm{ML}$ algorithm-deliver more insights into the specific imaging features which were important for the model's classification performance. In summary, solutions achieving better standardization or normalization resulting in better generalisability are an important condition to bring radiomics and AI into cardiac precision medicine with concomitant improved diagnostic approaches to CVDs. In addition, better interoperability of healthcare informatics systems should be achieved. Finally, the steadfast progression of AI approaches to clinical decision making represent an abrupt change from conventional medical reasoning, as such, it is essential to engage with the psychological impact of the ongoing digital transformation in order to facilitate the transition of medical practice in line with advancing technologies. The extensive and encouraging work reviewed in this article above pursues one common goal for the future of cardiovascular medicine: to pave the way toward better diagnosis and precision medicine in cardiology. The application of AI to cardiology holds the promise to revolutionize individual disease monitoring and treatment (93), thus overcoming the currently used "one size fits all" approach derived from large clinical studies.

\section{AUTHOR CONTRIBUTIONS}

CM-I performed the literature search and its categorization, developed statistics, wrote the diagnostics literature section, and coordinated the remaining sections. VC wrote the machine learning section and contributed to the remaining sections. CI wrote the data section and designed the figures. KL supervised the manuscript development, wrote the abstract and the introduction, and reviewed all sections. ZR-E, BB, and SP contributed to drafting and critical appraisal of the manuscript. All authors reviewed the manuscript.

\section{FUNDING}

This work was partly funded by the European Union's Horizon 2020 research and innovation programme under grant agreement No 825903 (euCanSHare project). SP acts as a paid consultant to Circle Cardiovascular Imaging Inc., Calgary, Canada and Servier. SP acknowledges support from the National Institute for Health Research (NIHR) Cardiovascular Biomedical Research Centre at Barts, from the SmartHeart EPSRC programme grant (EP/P001009/1) and the London Medical Imaging and AI Centre for Value-Based Healthcare. $\mathrm{SP}$ and KL acknowledge support from the CAP-AI programme, London's first AI enabling programme focused on stimulating growth in the capital's AI sector. ZR-E was supported by a British Heart Foundation Clinical Research Training Fellowship (FS/17/81/33318). 


\section{REFERENCES}

1. Wilkins E, Wilson L, Wickramasinghe K, Bhatnagar P, Leal J, LuengoFernandez R, et al. European Cardiovascular Disease Statistics 2017. Brussels: European Heart Network (2017).

2. Ritchie H, Roser M. Our world in data. In: Causes of Death (2018). Retrieved from: https://ourworldindata.org/causes-of-death.

3. Leiner $\mathrm{T}$, Rueckert $\mathrm{D}$, Suinesiaputra A, Baeßler B, Nezafat R, Išgum $\mathrm{I}$, et al. Machine learning in cardiovascular magnetic resonance: basic concepts and applications. $J$ Cardiovasc Magn Resonan. (2019) 21:61. doi: 10.1186/s12968-0190575-y

4. Khened M, Alex V, Krishnamurthi G. Densely connected fully convolutional network for short-axis cardiac cine MR image segmentation and heart diagnosis using random forest. In: International Workshop on Statistical Atlases and Computational Models of the Heart. Quebec City, QC: Springer (2017). p. 140-51.

5. Chen $\mathrm{R}, \mathrm{Lu} \mathrm{A}$, Wang J, Ma X, Zhao L, Wu W, et al. Using machine learning to predict one-year cardiovascular events in patients with severe dilated cardiomyopathy. Eur J Radiol. (2019). 117:178-83. doi: 10.1016/j.ejrad.2019.06.004

6. Juarez-Orozco LE, Knol RJ, Sanchez-Catasus CA, Martinez-Manzanera O, van der Zant FM, Knuuti J. Machine learning in the integration of simple variables for identifying patients with myocardial ischemia. J Nucl Cardiol. (2018). doi: 10.1007/s12350-018-1304-x. [Epub ahead of print].

7. Mantilla J, Garreau M, Bellanger JJ, Paredes JL. Machine learning techniques for LV wall motion classification based on spatio-temporal profiles from cardiac cine MRI. In: 2013 12th International Conference on Machine Learning and Applications. Vol. 1. IEEE (2013). p. 167-72.

8. Bagher-Ebadian H, Soltanian-Zadeh H, Setayeshi S, Smith ST. Neural network and fuzzy clustering approach for automatic diagnosis of coronary artery disease in nuclear medicine. IEEE Trans Nucl Sci. (2004) 51:184-92. doi: 10.1109/TNS.2003.823047

9. Moreno A, Rodriguez J, Martínez F. Regional multiscale motion representation for cardiac disease prediction. In: 2019 XXII Symposium on Image, Signal Processing and Artificial Vision (STSIVA). Bucaramanga, CO: IEEE (2019). p. 1-5.

10. Zheng Q, Delingette $H$, Ayache N. Explainable cardiac pathology classification on cine MRI with motion characterization by semisupervised learning of apparent flow. Med Image Anal. (2019) 56:80-95. doi: 10.1016/j.media.2019.06.001

11. Lambin P, Rios-Velazquez E, Leijenaar R, Carvalho S, Van Stiphout RG, Granton P, et al. Radiomics: extracting more information from medical images using advanced feature analysis. Eur J Cancer. (2012) 48:441-6. doi: 10.1016/j.ejca.2011.11.036

12. Kumar V, Gu Y, Basu S, Berglund A, Eschrich SA, Schabath MB, et al. Radiomics: the process and the challenges. Magn Reson Imaging. (2012) 30:1234-48. doi: 10.1016/j.mri.2012.06.010

13. Aerts HJ, Velazquez ER, Leijenaar RT, Parmar C, Grossmann P, Carvalho $\mathrm{S}$, et al. Decoding tumour phenotype by noninvasive imaging using a quantitative radiomics approach. Nat Commun. (2014) 5:4006. doi: 10.1038/ncomms5644

14. Cetin I, Sanroma G, Petersen SE, Napel S, Camara O, Ballester MAG, et al. A radiomics approach to computer-aided diagnosis with cardiac cine-MRI. In: International Workshop on Statistical Atlases and Computational Models of the Heart. Quebec City, QC: Springer (2017). p. 82-90.

15. Neisius U, El-Rewaidy H, Nakamori S, Rodriguez J, Manning WJ, Nezafat R. Radiomic analysis of myocardial native T1 imaging discriminates between hypertensive heart disease and hypertrophic cardiomyopathy. JACC Cardiovasc Imaging. (2019) 12:1946-1954. doi: 10.1016/j.jcmg.2018. 11.024

16. Betancur J, Hu LH, Commandeur F, Sharir T, Einstein AJ, Fish MB, et al. Deep learning analysis of upright-supine high-efficiency spect myocardial perfusion imaging for prediction of obstructive coronary artery disease: a multicenter study. J Nucl Med. (2019) 60:664-70. doi: 10.2967/jnumed.118.213538

17. Wolterink JM, Leiner $\mathrm{T}$, de Vos BD, van Hamersvelt RW, Viergever MA, Išgum I. Automatic coronary artery calcium scoring in cardiac CT angiography using paired convolutional neural networks. Med Image Anal. (2016) 34:123-36. doi: 10.1016/j.media.2016.04.004

18. Lu A, Dehghan E, Veni G, Moradi M, Syeda-Mahmood T. Detecting anomalies from echocardiography using multi-view regression of clinical measurements. In: 2018 IEEE 15th International Symposium on Biomedical Imaging (ISBI 2018). IEEE (2018). p. 1504-8.

19. Kusunose K, Abe T, Haga A, Fukuda D, Yamada H, Harada M, et al. A deep learning approach for assessment of regional wall motion abnormality from echocardiographic images. JACC Cardiovasc Imaging. (2019). [Epub ahead of print].

20. Arsanjani R, Xu Y, Dey D, Vahistha V, Shalev A, Nakanishi R, et al. Improved accuracy of myocardial perfusion SPECT for detection of coronary artery disease by machine learning in a large population. J Nucl Cardiol. (2013) 20:553-62. doi: 10.1007/s12350-013-9706-2

21. Baeßler B, Luecke C, Lurz J, Klingel K, Das A, von Roeder M, et al. Cardiac MRI and texture analysis of myocardial T1 and T2 maps in myocarditis with acute versus chronic symptoms of heart failure. Radiology. (2019) 292:608-17. doi: 10.1148/radiol.2019190101

22. Conforti D, Guido R. Kernel-based support vector machine classifiers for early detection of myocardial infarction. Optimizat Methods Softw. (2005) 20:401-13. doi: 10.1080/105567805123313 18164

23. Arsanjani R, Xu Y, Dey D, Fish M, Dorbala S, Hayes S, et al. Improved accuracy of myocardial perfusion SPECT for the detection of coronary artery disease using a support vector machine algorithm. J Nucl Med. (2013) 54:549-55. doi: 10.2967/jnumed.112.111542

24. Ciecholewski M. Support vector machine approach to cardiac SPECT diagnosis. In: International Workshop on Combinatorial Image Analysis. Madrid: Springer (2011). p. 432-43.

25. Berikol GB, Yildiz O, Özcan IT. Diagnosis of acute coronary syndrome with a support vector machine. J Med Syst. (2016) 40:84. doi: 10.1007/s10916-016-0432-6

26. Borkar S, Annadate M. Supervised machine learning algorithm for detection of cardiac disorders. In: 2018 Fourth International Conference on Computing Communication Control and Automation (ICCUBEA). Pune: IEEE (2018). p. $1-4$.

27. Wong KC, Tee M, Chen M, Bluemke DA, Summers RM, Yao J. Regional infarction identification from cardiac CT images: a computer-aided biomechanical approach. Int J Comput Assist Radiol Surg. (2016) 11:1573-83. doi: 10.1007/s11548-016-1404-5

28. Baeßler B, Mannil M, Maintz D, Alkadhi H, Manka R. Texture analysis and machine learning of non-contrast T1-weighted MR images in patients with hypertrophic cardiomyopathy-preliminary results. Eur J Radiol. (2018) 102:61-7. doi: 10.1016/j.ejrad.2018.03.013

29. Tabassian M, Alessandrini M, Herbots L, Mirea O, Pagourelias ED, Jasaityte R, et al. Machine learning of the spatio-temporal characteristics of echocardiographic deformation curves for infarct classification. Int J Cardiovasc Imaging. (2017) 33:1159-67. doi: 10.1007/s10554-0171108-0

30. Išgum I, van Ginneken B, Olree M. Automatic detection of calcifications in the aorta from CT scans of the abdomen 1: 3D computer-aided diagnosis. Acad Radiol. (2004) 11:247-57. doi: 10.1016/S1076-6332(03)00673-1

31. Bruse JL, Zuluaga MA, Khushnood A, McLeod K, Ntsinjana HN, Hsia TY, et al. Detecting clinically meaningful shape clusters in medical image data: metrics analysis for hierarchical clustering applied to healthy and pathological aortic arches. IEEE Trans Biomed Eng. (2017) 64:2373-83. doi: 10.1109/TBME.2017.2655364

32. Wojnarski CM, Roselli EE, Idrees JJ, Zhu Y, Carnes TA, Lowry AM, et al. Machine-learning phenotypic classification of bicuspid aortopathy. J Thorac Cardiovasc Surg. (2018) 155:461-9. doi: 10.1016/j.jtcvs.2017.08.123

33. Tsai DY, Lee Y, Sekiya M, Ohkubo M. Medical image classification using genetic-algorithm based fuzzy-logic approach. J Electr Imaging. (2004) 13:780-9. doi: 10.1117/1.1786607

34. Nakajima K, Kudo T, Nakata T, Kiso K, Kasai T, Taniguchi Y, et al. Diagnostic accuracy of an artificial neural network compared with statistical quantitation of myocardial perfusion images: a Japanese multicenter study. Eur J Nucl Med Mol Imaging. (2017) 44:2280-9. doi: 10.1007/s00259-017-3834-x 
35. Nakajima K, Okuda $\mathrm{K}$, Watanabe $\mathrm{S}$, Matsuo S, Kinuya S, Toth $\mathrm{K}$, et al. Artificial neural network retrained to detect myocardial ischemia using a Japanese multicenter database. Ann Nucl Med. (2018) 32:303-10. doi: 10.1007/s12149-018-1247-y

36. Zhang J, Gajjala S, Agrawal P, Tison GH, Hallock LA, BeussinkNelson L, et al. Fully automated echocardiogram interpretation in clinical practice: feasibility and diagnostic accuracy. Circulation. (2018) 138:1623-35. doi: 10.1161/CIRCULATIONAHA.118.034338

37. Madani A, Ong JR, Tibrewal A, Mofrad MR. Deep echocardiography: data-efficient supervised and semi-supervised deep learning towards automated diagnosis of cardiac disease. npj Digit Med. (2018) 1:59. doi: 10.1038/s41746-018-0065-x

38. Cetin I, Petersen SE, Napel S, Camara O, Ballester MÁG, Lekadir K. A radiomics approach to analyze cardiac alterations in hypertension. In: 2019 IEEE 16th International Symposium on Biomedical Imaging (ISBI 2019). Venice: IEEE (2019). p. 640-3.

39. Nakada A, Shiozaki A, Hirano Y, Uehara H, Masuyama T. Using neural networks in color kinesis image process to automate diagnosis of cardiac disease. Electr Commun Jpn (Part II: Electr). (2006) 89:46-54. doi: $10.1002 /$ ecjb. 20245

40. Ungru K, Tenbrinck D, Jiang X, Stypmann J. Automatic classification of left ventricular wall segments in small animal ultrasound imaging. Comput Methods Progr Biomed. (2014) 117:2-12. doi: 10.1016/j.cmpb.2014.06.015

41. Agani N, Abu-Bakar S, Salleh SS. Application of texture analysis in echocardiography images for myocardial infarction tissue. Jurnal Teknologi. (2007) 46:61-76. doi: 10.11113/jt.v46.295

42. Sudarshan VK, Acharya UR, Ng E, San Tan R, Chou SM, Ghista DN. An integrated index for automated detection of infarcted myocardium from cross-sectional echocardiograms using texton-based features (Part 1). Comput Biol Med. (2016) 71:231-40. doi: 10.1016/j.compbiomed.2016. 01.028

43. Vidya KS, Ng E, Acharya UR, Chou SM, San Tan R, Ghista DN. Computeraided diagnosis of myocardial infarction using ultrasound images with DWT, GLCM and HOS methods: a comparative study. Comput Biol Med. (2015) 62:86-93. doi: 10.1016/j.compbiomed.2015.03.033

44. Baeßler B, Mannil M, Oebel S, Maintz D, Alkadhi H, Manka R. Subacute and chronic left ventricular myocardial scar: accuracy of texture analysis on nonenhanced cine MR images. Radiology. (2017) 286:103-12. doi: $10.1148 /$ radiol.2017170213

45. Larroza A, López-Lereu MP, Monmeneu JV, Gavara J, Chorro FJ, Bodí V, et al. Texture analysis of cardiac cine magnetic resonance imaging to detect nonviable segments in patients with chronic myocardial infarction. Med Phys. (2018) 45:1471-80. doi: 10.1002/mp.12783

46. Zhang N, Yang G, Gao Z, Xu C, Zhang Y, Shi R, et al. Deep learning for diagnosis of chronic myocardial infarction on nonenhanced cardiac Cine MRI. Radiology. (2019) 291:606-17. doi: 10.1148/radiol.20191 82304

47. Mannil M, von Spiczak J, Manka R, Alkadhi H. Texture analysis and machine learning for detecting myocardial infarction in noncontrast lowdose computed tomography: unveiling the invisible. Investigat Radiol. (2018) 53:338-43. doi: 10.1097/RLI.0000000000000448

48. Mannil M, von Spiczak J, Muehlematter UJ, Thanabalasingam A, Keller DI, Manka R, et al. Texture analysis of myocardial infarction in CT: comparison with visual analysis and impact of iterative reconstruction. Eur J Radiol. (2019) 113:245-50. doi: 10.1016/j.ejrad.2019.02.037

49. Gopalakrishnan V, Menon PG, Madan S. cMRI-BED: a novel informatics framework for cardiac MRI biomarker extraction and discovery applied to pediatric cardiomyopathy classification. Biomed Eng Online. (2015) 14:S7. doi: 10.1186/1475-925X-14-S2-S7

50. Narula S, Shameer K, Omar AMS, Dudley JT, Sengupta PP. Machinelearning algorithms to automate morphological and functional assessments in 2D echocardiography. J Am Coll Cardiol. (2016) 68:2287-95. doi: 10.1016/j.jacc.2016.08.062

51. Bernard O, Lalande A, Zotti C, Cervenansky F, Yang X, Heng PA, et al. Deep learning techniques for automatic MRI cardiac multi-structures segmentation and diagnosis: is the problem solved? IEEE Trans Med Imaging. (2018) 37:2514-25. doi: 10.1109/TMI.2018.2837502
52. Wolterink JM, Leiner T, Viergever MA, Išgum I. Automatic segmentation and disease classification using cardiac cine MR images. In: International Workshop on Statistical Atlases and Computational Models of the Heart. Quebec City, QC: Springer (2017). p. 101-10.

53. Isensee F, Jaeger PF, Full PM, Wolf I, Engelhardt S, Maier-Hein KH. Automatic cardiac disease assessment on cine-MRI via time-series segmentation and domain specific features. In: International Workshop on Statistical Atlases and Computational Models of the Heart. Quebec City, QC: Springer (2017). p. 120-9.

54. Snaauw G, Gong D, Maicas G, van den Hengel A, Niessen WJ, Verjans $\mathrm{J}$, et al. End-to-end diagnosis and segmentation learning from cardiac magnetic resonance imaging. In: 2019 IEEE 16th International Symposium on Biomedical Imaging (ISBI 2019). Venice: IEEE (2019). p. 802-5.

55. Biffi C, Oktay O, Tarroni G, Bai W, De Marvao A, Doumou G, et al. Learning interpretable anatomical features through deep generative models: application to cardiac remodeling. In: International Conference on Medical Image Computing and Computer-Assisted Intervention. Granada: Springer (2018). p. 464-71.

56. Puyol-Antón E, Ruijsink B, Gerber B, Amzulescu MS, Langet H, De Craene $\mathrm{M}$, et al. Regional multi-view learning for cardiac motion analysis: application to identification of dilated cardiomyopathy patients. IEEE Trans Biomed Eng. (2018) 66:956-66. doi: 10.1109/TBME.2018.2865669

57. Neisius U, Myerson L, Fahmy AS, Nakamori S, El-Rewaidy H, Joshi G, et al. Cardiovascular magnetic resonance feature tracking strain analysis for discrimination between hypertensive heart disease and hypertrophic cardiomyopathy. PLoS ONE. (2019) 14:e0221061. doi: 10.1371/journal.pone.0221061

58. Kukar M, Kononenko I, Grošelj C, Kralj K, Fettich J. Analysing and improving the diagnosis of ischaemic heart disease with machine learning. Artif Intell Med. (1999) 16:25-50. doi: 10.1016/S0933-3657(98)00063-3

59. Kurgan LA, Cios KJ, Tadeusiewicz R, Ogiela M, Goodenday LS. Knowledge discovery approach to automated cardiac SPECT diagnosis. Artif Intell Med. (2001) 23:149-69. doi: 10.1016/S0933-3657(01)00082-3

60. Guner LA, Karabacak NI, Akdemir OU, Karagoz PS, Kocaman SA, Cengel A, et al. An open-source framework of neural networks for diagnosis of coronary artery disease from myocardial perfusion SPECT. J Nucl Cardiol. (2010) 17:405-13. doi: 10.1007/s12350-010-9207-5

61. Shibutani T, Nakajima K, Wakabayashi H, Mori H, Matsuo S, Yoneyama $\mathrm{H}$, et al. Accuracy of an artificial neural network for detecting a regional abnormality in myocardial perfusion SPECT. Ann Nucl Med. (2019) 33:86-92. doi: 10.1007/s12149-018-1306-4

62. Han D, Lee JH, Rizvi A, Gransar H, Baskaran L, Schulman-Marcus J, et al. Incremental role of resting myocardial computed tomography perfusion for predicting physiologically significant coronary artery disease: a machine learning approach. J Nucl Cardiol. (2018) 25:223-33. doi: 10.1007/s12350-017-0834-y

63. Coenen A, Kim YH, Kruk M, Tesche C, De Geer J, Kurata A, et al. Diagnostic accuracy of a machine-learning approach to coronary computed tomographic angiography-based fractional flow reserve: result from the MACHINE consortium. Circulation. (2018) 11:e007217. doi: 10.1161/CIRCIMAGING.117.007217

64. Išgum I, Rutten A, Prokop M, van Ginneken B. Detection of coronary calcifications from computed tomography scans for automated risk assessment of coronary artery disease. Med Phys. (2007) 34:1450-61. doi: $10.1118 / 1.2710548$

65. Wolterink JM, Leiner T, Takx RA, Viergever MA, Išgum I. An automatic machine learning system for coronary calcium scoring in clinical non-contrast enhanced, ECG-triggered cardiac CT. In: Medical Imaging 2014: ComputerAided Diagnosis, Vol. 9035. San Diego, CA: International Society for Optics and Photonics (2014). p. 90350E. doi: 10.1117/12.2042226

66. Kolossváry M, Karády J, Szilveszter B, Kitslaar P, Hoffmann U, Merkely B, et al. Radiomic features are superior to conventional quantitative computed tomographic metrics to identify coronary $\begin{array}{llll}\text { plaques with napkin-ring sign. Circulation. (2017) 10:e0 } & \end{array}$ 06843. doi: 10.1161/CIRCIMAGING.117.006843

67. Zreik M, van Hamersvelt RW, Wolterink JM, Leiner T, Viergever MA, Išgum I. A recurrent $\mathrm{CNN}$ for automatic detection and classification of coronary artery 
plaque and stenosis in coronary CT angiography. IEEE Trans Med Imaging. (2018) 38:1588-1598. doi: 10.1109/TMI.2018.2883807

68. Elalfi A, Eisa M, Ahmed H. Artificial neural networks in medical images for diagnosis heart valve diseases. Int J Comput Sci Issues. (2013) 10:83. Available online at: https://www.ijcsi.org

69. Moghaddasi H, Nourian S, Nourian S. Automatic assessment of mitral regurgitation severity based on extensive textural features on 2D echocardiography videos. Comput Biol Med. (2016) 73:47-55. doi: 10.1016/j.compbiomed.2016.03.026

70. Tabassian M, Sunderji I, Erdei T, Sanchez-Martinez S, Degiovanni A, Marino $\mathrm{P}$, et al. Diagnosis of heart failure with preserved ejection fraction: machine learning of spatiotemporal variations in left ventricular deformation. J Am Soc Echocardiogr. (2018) 31:1272-84. doi: 10.1016/j.echo.2018.07.013

71. Shah SJ, Katz DH, Selvaraj S, Burke MA, Yancy CW, Gheorghiade $\mathrm{M}$, et al. Phenomapping for novel classification of heart failure with preserved ejection fraction. Circulation. (2015) 131:269-79. doi: 10.1161/CIRCULATIONAHA.114.010637

72. Cikes M, Sanchez-Martinez S, Claggett B, Duchateau N, Piella G, Butakoff $\mathrm{C}$, et al. Machine learning-based phenogrouping in heart failure to identify responders to cardiac resynchronization therapy. Eur J Heart Fail. (2019) 21:74-85. doi: 10.1002/ejhf.1333

73. Afshin M, Ayed IB, Punithakumar K, Law M, Islam A, Goela A, et al. Regional assessment of cardiac left ventricular myocardial function via MRI statistical features. IEEE Trans Med Imaging. (2013) 33:481-94. doi: 10.1109/TMI.2013.2287793

74. Ibánez B, James S, Agewall S, Antunes MJ, Bucciarelli-Ducci C, Bueno H, et al. 2017 ESC Guidelines for the management of acute myocardial infarction in patients presenting with ST-segment elevation. Revista Espanola de Cardiologia (English ed). (2017) 70:1082. doi: 10.1016/j.rec.2017.11.010

75. Park JE, Kim HS. Radiomics as a quantitative imaging biomarker: practical considerations and the current standpoint in neuro-oncologic studies. Nucl Med Mol Imaging. (2018) 52:99-108. doi: 10.1007/s13139-017-0512-7

76. Zwanenburg A, Leger S, Vallières M, Löck S. Image biomarker standardisation initiative. arXiv:161207003. (2016).

77. Balagurunathan Y, Kumar V, Gu Y, Kim J, Wang H, Liu Y, et al. Test-retest reproducibility analysis of lung CT image features. J Digit Imaging. (2014) 27:805-23. doi: 10.1007/s10278-014-9716-x

78. Lubner MG, Smith AD, Sandrasegaran K, Sahani DV, Pickhardt PJ. CT texture analysis: definitions, applications, biologic correlates, and challenges. Radiographics. (2017) 37:1483-503. doi: 10.1148/rg.2017170056

79. Mackin D, Fave X, Zhang L, Fried D, Yang J, Taylor B, et al. Measuring CT scanner variability of radiomics features. Investigat Radiol. (2015) 50:757. doi: 10.1097/RLI.0000000000000180

80. Nyflot MJ, Yang F, Byrd D, Bowen SR, Sandison GA, Kinahan PE. Quantitative radiomics: impact of stochastic effects on textural feature analysis implies the need for standards. J Med Imaging. (2015) 2:041002. doi: 10.1117/1.JMI.2.4.041002

81. Shafiq-ul Hassan M, Zhang GG, Latifi K, Ullah G, Hunt DC, Balagurunathan $\mathrm{Y}$, et al. Intrinsic dependencies of CT radiomic features on voxel size and number of gray levels. Med Phys. (2017) 44:1050-62. doi: 10.1002/mp.12123

82. Zhao B, Tan Y, Tsai WY, Qi J, Xie C, Lu L, et al. Reproducibility of radiomics for deciphering tumor phenotype with imaging. Sci Rep. (2016) 6:23428. doi: $10.1038 /$ srep23428

83. Zhao B, Tan Y, Tsai WY, Schwartz LH, Lu L. Exploring variability in CT characterization of tumors: a preliminary phantom study. Transl oncol. (2014) 7:88. doi: 10.1593/tlo. 13865

84. Hinzpeter R, Wagner MW, Wurnig MC, Seifert B, Manka R, Alkadhi H. Texture analysis of acute myocardial infarction with CT: first experience study. PLoS ONE. (2017) 12:e0186876. doi: 10.1371/journal.pone.0186876

85. Collewet G, Strzelecki M, Mariette F. Influence of MRI acquisition protocols and image intensity normalization methods on texture classification. Magn Reson Imaging. (2004) 22:81-91. doi: 10.1016/j.mri.2003.09.001
86. Mayerhoefer ME, Szomolanyi P, Jirak D, Berg A, Materka A, Dirisamer A, et al. Effects of magnetic resonance image interpolation on the results of texture-based pattern classification: a phantom study. Investigat Radiol. (2009) 44:405-11. doi: 10.1097/RLI.0b013e3181a50a66

87. Saha A, Harowicz MR, Mazurowski MA. Breast cancer MRI radiomics: an overview of algorithmic features and impact of inter-reader variability in annotating tumors. Med Phys. (2018) 45:3076-85. doi: 10.1002/mp. 12925

88. Baeßler B, Weiss K, dos Santos DP. Robustness and reproducibility of radiomics in magnetic resonance imaging: a phantom study. Investigat Radiol. (2019) 54:221-8. doi: 10.1097/RLI.0000000000000530

89. Gallardo-Estrella L, Lynch DA, Prokop M, Stinson D, Zach J, Judy PF, et al. Normalizing computed tomography data reconstructed with different filter kernels: effect on emphysema quantification. Eur Radiol. (2016) 26:478-86. doi: $10.1007 / \mathrm{s} 00330-015-3824-y$

90. Jin $\mathrm{H}, \mathrm{Heo} \mathrm{C}$, Kim JH. Deep learning-enabled accurate normalization of reconstruction kernel effects on emphysema quantification in lowdose CT. Phys Med Biol. (2019) 64:135010. doi: 10.1088/1361-6560/ ab28a1

91. Samala RK, Chan HP, Hadjiiski L, Paramagul C, Helvie MA, Neal CH. Homogenization of breast MRI across imaging centers and feature analysis using unsupervised deep embedding. In: Medical Imaging 2019: ComputerAided Diagnosis, Vol. 10950. San Diego, CA: International Society for Optics and Photonics (2019). p. 1095020. doi: 10.1117/12.2512209

92. Dewey BE, Zhao C, Reinhold JC, Carass A, Fitzgerald KC, Sotirchos ES, et al. DeepHarmony: a deep learning approach to contrast harmonization across scanner changes. Magn Reson Imaging. (2019) 64:160-70. doi: 10.1016/j.mri.2019.05.041

93. Leopold JA, Loscalzo J. Emerging role of precision medicine in cardiovascular disease. Circulat Res. (2018) 122:1302-15. doi: 10.1161/CIRCRESAHA.117.310782

94. Rohé MM, Duchateau N, Sermesant M, Pennec X. Combination of polyaffine transformations and supervised learning for the automatic diagnosis of LV infarct. In: Statistical Atlases and Computational Models of the Heart. Munich: Springer (2015). p. 190-8.

95. Lekadir K, Albà X, Pereañez M, Frangi AF. Statistical shape modeling using partial least squares: application to the assessment of myocardial infarction. In: Statistical Atlases and Computational Models of the Heart. Munich: Springer (2015). p. 130-9.

96. Sacha JP, Goodenday LS, Cios KJ. Bayesian learning for cardiac SPECT image interpretation. Artif Intell Med. (2002) 26:109-43. doi: 10.1016/S0933-3657(02)00055-6

97. To C, Pham TD. Analysis of cardiac imaging data using decision tree based parallel genetic programming. In: 2009 Proceedings of 6th International Symposium on Image and Signal Processing and Analysis. Salzburg: IEEE (2009). p. 317-20.

98. Zhang L, Wahle A, Chen Z, Lopez JJ, Kovarnik T, Sonka M. Predicting locations of high-risk plaques in coronary arteries in patients receiving statin therapy. IEEE Trans Med Imaging. (2017) 37:151-61. doi: 10.1109/TMI.2017.2725443

Conflict of Interest: The authors declare that the research was conducted in the absence of any commercial or financial relationships that could be construed as a potential conflict of interest.

Copyright (C) 2020 Martin-Isla, Campello, Izquierdo, Raisi-Estabragh, Baeßler, Petersen and Lekadir. This is an open-access article distributed under the terms of the Creative Commons Attribution License (CC BY). The use, distribution or reproduction in other forums is permitted, provided the original author(s) and the copyright owner(s) are credited and that the original publication in this journal is cited, in accordance with accepted academic practice. No use, distribution or reproduction is permitted which does not comply with these terms. 